\title{
Erzähltheoretische Grundlagen für uRE
}

Nachdem im vorausgegangenen Kapitel der Untersuchungsgegenstand im Fachbereich der Textlinguistik verortet und anhand dessen beschrieben wurde, führe ich die Bestimmung der erhobenen uRE nun im erzähltheoretischen Bereich fort. In diesem Kapitel geht es also darum, (1) die uRE unter einer narratologischen Perspektive zu betrachten und (2) den narratologischen Rahmen vorzustellen, in dem sich die uRE bewegen. Einleitend greife ich Termini auf (Abschn. 5.1), die aufgrund ihrer vielfältigen Anwendung im Rahmen dieser Arbeit genauer gefasst werden müssen. Die anschließenden Kapitel sind bereits die Basis für das entstandene Verortungsschema. Ich werde vier erzähltheoretische Merkmale vorstellen, die für die Untersuchungsobjekte konstitutiv sind. Die Merkmale stammen sowohl aus der linguistischen als auch der literaturwissenschaftlichen Erzählforschung. Sie stellen die inhaltlichen Grundmerkmale für das datengeleitete Erzählverständnis, auf das ich mich stütze, um die uRE als Untersuchungsgegenstand der Erzählforschung zu legitimieren (Abschn. 5.2). Das daran anschließende Kapitel erweitert die inhaltlichen Merkmale um charakteristisch formale Grundkennzeichen des vorgefundenen Materials (Abschn. 5.3). Die zwei Kapitel münden in ein Verortungsschema, das die beschriebenen Kennzeichen der uRE zusammenfasst. Ich werde explizieren, wie ich das Schema für den Umgang mit der heterogenen Datenlage nutze (Abschn. 5.4). Meine Vorgehensweise verdeutlicht, dass ich die Linguistik und die Literaturwissenschaft als eigene Expertisen mit eigenen Schwerpunkten sowie Blickwinkeln ansehe. Nünning/Nünning (2002: 19) plädieren im Sinne der Narratologie dafür, dass die verschiedenen Fachrichtungen (Literatur, Linguistik, Psychologie, Geschichte u. a.) nicht nur koexistieren, sondern voneinander lernen sollten, da Schlüsselbegriffe der narratologischen Forschung (z. B. Ereignis, Erzählperspektive) für alle Disziplinen relevant sind. Dabei hat die Literaturwissenschaft aus ihrer langjährigen Beschäftigung mit der Belletristik ein ,umfangreiches Beschreibungsinventar 
entwickelt, um die mehrschichtigen Strukturen von Erzählungen und die Perspektiven zu erfassen“ (Adamzik 2016: 283). Demgegenüber fokussiert die Linguistik heute verstärkt nicht-literarische Formen, insbesondere mündliche Alltagserzählungen (Gülich 2008: 403). Gülich (2008) sieht darin eine Arbeitsteilung zwischen den Disziplinen. Da sich das Korpus gerade dadurch auszeichnen, dass die Erzähler/innen Merkmale aus beiden Fachbereichen nutzen (z. B. Abschn. 9.2.4.2), ist es m. E. gewinnbringend, aus den jeweiligen Disziplinen das herauszugreifen, was die entsprechende uRE erfordert. Meiner These folgend sehe ich in den uRE narrative Praktiken, die aus der funktionellen Orientierung zur (S)D resultieren. Deutlich wird das an Ehlichs (1980) Aussage über ,funktionalisiertes Erzählen“ im institutionellen Kontext:

„Die Funktion, unter die das Erzählen subsumiert wird, wird von außen - von den für die Institution charakteristischen Zwecken her, die die Handelnden erfüllen wollen an das Erzählen herangetragen“ (ebd. 1980: 137).

Die Herausforderung, die uRE in einen erzähltheoretischen Zusammenhang zu stellen, entsteht durch den Leitgedanken dieser Arbeit. Es geht darum, sich an den vorgefundenen Erscheinungsformen von Erzählungen zu orientieren, die eine imagefördernden (S)D kommunizieren, und nicht die Erzählungen nach einem spezifischen Erzählmodell auszuwählen, um dann daraus S(D) zu entnehmen. Die konsequente Ausrichtung an dem existierenden Datenmaterial hat zur Folge, dass je nach narrativem Vermittlungsvorkommen verschiedene erzähltheoretische Ansätze und Ausrichtungen relevant werden.

\subsection{Grundbegriffe: das narrative „Allerlei“}

Das große disziplinübergreifende Interesse an dem Untersuchungsgegenstand ,Erzählen“ hat zu einer Begriffsvielfalt und inflationären Verwendung verschiedener Termini geführt, die ich einleitend differenzieren werde. Aufgrund der interdisziplinären Ausrichtung der Arbeit ist es notwendig, ihre Verwendung zu benennen sowie eine Minimalbedingung für das ,Narrative“ voranzustellen. Sie ist die Grundlage des Verortungsschemas.

Insbesondere der Begriff ,Narrativität' ist im disziplinübergreifenden Fachdiskurs omnipräsent.,Narrativität' bezieht sich darauf, ,eine Geschichte zu [...] repräsentieren“ (Aumüller 2012: 9, Hervorhebung i. O.). Ursächlich für die Begriffsbildung sind nicht nur ,angloamerikanische[] und französische[] Theorieimpulse“ (ebd., engl. narrativity). Vorrangig geht es um eine Abgrenzung 
von der umgangssprachlichen Verwendung des Erzählbegriffs. Denn - so Ehlich (1980b: 19; 1983: 128 f.) und Kindt (1997) - in der Alltagskommunikation kann „[i]m unspezifischen Sinne [...] nahezu jede Art der Mitteilung als Erzählen eingestuft werden“" (Kindt 1997: 7). Beispielsweise formulieren wir mit dem Begriff Fragen, die eine Erklärung - also eine vollkommen andere Sprechhandlung erfordern (kannst Du mir mal erzählen, warum du zu spät kommst? (vgl. Ehlich 1983a)). Da unter Narrativität verstanden wird, eine Geschichte zu repräsentieren, ist ebenso das in der Alltagssprache bezeichnende Merkmal ,Sprachlichkeit" unwirksam. Das führt dazu, dass der Ausdruck ,Narrativität ' auch andere Repräsentationsoptionen einschließt und umfassend anwendbar ist. Die Bedeutung des Ausdrucks ,Narration“ ist dagegen von der englischen Bezeichnung ,narrative“ übernommen worden und bedeutet das deutsche Pendant; nämlich ,Erzählung'. (Kuhn 2013: 64) „[D]as dazugehörige Adjektiv ist narrativ“ (ebd., Hervorhebung, U.A.) und bedeutet ,erzählerisch`.

In diesem Zusammenhang muss auch das derzeit allgegenwärtige ,Narrativ erwähnt werden, das als Modewort (Zifonun 2017: 1) im öffentlichen Diskurs seine Spuren hinterlässt ${ }^{1}$. Dabei handelt es sich um eine Entlehnung aus dem englischen in den deutschen Sprachgebrauch, die bisher noch nicht im Duden verzeichnet ist. Im Gegensatz zu dem englischen Adjektiv ,narrative', dem im Englischen das gleichlautende Substantiv zugehört, ist das im Deutschen nicht der Fall. Seit dem 19. Jh. findet der Begriff ,Narrativ' im narratologischen Diskurs insbesondere in den Literaturwissenschaften unter der Bedeutung ,Geschichte' Verwendung. Im Rahmen des Storytelling für Unternehmen macht der Begriff in dem Kompositum ,Basis-Narrativ“ Karriere. Im Bereich der Public Relations wird darunter ein abstraktes, „kulturell überliefertes Grundmuster“ (Stücheli-Herlach/Perrin 2013: 31) verstanden, das die Rezipient/innen aufgrund der Komponenten und ihrer Zusammensetzung themenunabhängig wiedererkennen. Ein bekanntes Basis-Narrativ ${ }^{2}$ ist in diesem Kontext beispielsweise die Heldenreise $^{3}$. In der linguistischen Erzählforschung spielt das ,Narrativ ${ }^{6}$ erst seit Kurzem im Rahmen der Diskursanalyse eine Rolle: Es erfasst narrative

${ }^{1}$ Z. B. in einem Artikel von Heine für die Welt Hinz und Kunz schwafeln heutzutage vom „Narrativ“ (Heine, Welt online, 2016-11-13) oder von Kniebe für die Süddeutschen Zeitung Modewort „Narrativ“ (Kniebe, SZ.de, 2017-08-15). (vgl. Zifonun 2017)

${ }^{2}$ Aus der Perspektive der Kommunikationswissenschaften spricht Krüger (2015) mit Bezug zum Corporate Storytelling von Frames, die spezifische Handlungs- und Sinnstrukturen bereitstellen, die mithilfe einer ,implizite[n] oder latente[n] Tiefenstruktur eine bestimmte Interpretation der Geschichte naheleg[en]“ (ebd. 2015: 124).

${ }^{3}$ Sie geht auf den Mythenforscher Joseph Campbell (1904) zurück. Campbell geht von einem universellen Erzählmodell aus, dem Archeplot der Heldenreise: Eine Person verlässt 
Muster in Diskursen und größeren Textkorpora. Bubenhofer et al. (2013) verstehen darunter ,sozial akzeptierte Interpretationsmuster, die unsere Wahrnehmung und Darstellung von Zusammenhängen ermöglichen und gleichzeitig begrenzen“ (Spieß/Tophinke 2018: 196). Nach einer Lesart, die den gesellschaftlichen und historischen Verhältnissen angepasst ist und auf den französischen Philosoph Lyotard zurückgeht, tritt das ,Narrativ “ an die Stelle der macrorécits ${ }^{4}$. In der Verwendung zeichnen sich Narrative nach Zifonun (2017: 1) in erster Linie durch Zugehörigkeitsattribute wie biblisches Narrativ, deutschnationales Narrativ oder feministisches Narrativ u. a. (ebd. 2017: 2) aus. Heutige Verwendungsweisen führen, so Zifonun, zu einer „Bedeutungsverschiebung ins Ungefähre und beförder[n] damit gleichzeitig, [...], seine Verwendung bei allen möglichen Gelegenheiten“ (ebd.). Daraus ergibt sich, dass der Terminus ,Narrativ“ im Fachdiskurs uneinheitlich angewandt und außerhalb des Fachdiskurses semantisch entleert ist.

Wenn wir uns dem Ausdruck, Erzählen“ zuwenden, dann zeigt sich hier eine ähnliche Problematik, da die Verwendung - auch im Fachdiskurs - mehrdeutig ist: Manche beziehen sich auf den Akt der Erzählens. Andere nutzen den Begriff für das Ergebnis des Erzählaktes und in der dritten Verwendungsoption dient er als Gattungsbegriff. (Köppe/Kindt 2014: 40) Ich folge in dem Wortfeld rund um ,Erzählen' der Terminologie und linguistischen Betrachtungsweise von Gülich (1976: 225 f.), die folgendermaßen differenziert:

- ,Erzählen“ ist die Bezeichnung für eine Sprechhandlung im Rahmen eines Kommunikationsprozesses.

- ,Erzähltext ${ }^{6}$ ist das, „was durch diese Sprachhandlung - mündlich oder schriftlich - geäußert wird“ (ebd. 1976: 226).

- ,Erzählung ' ist ein „Oberbegriff für die Textsorten-Klassen und als Gattungsbezeichnung für literarische Texte, wenn eine Festlegung auf ,Novelle‘ oder ,Kurzgeschichte“ usw. nicht möglich oder nicht zweckmäßig ist" (ebd.).

- ,Geschichte ${ }^{6}$ ist die Abfolge von realen (oder fiktiven) Ereignissen und Handlungen.

ihre gewohnte Umgebung und begibt sich auf ein Abenteuer, aus dem sie gestärkt zurückkehrt. Dabei durchläuft der Held verschiedene Herausforderungen und begegnet spezifischen „Archetypen“, die ihn unterstützen oder sein Vorhaben zum Scheitern bringen möchten. In Abschn. 7.2.2.1 wende ich die Archetypen auf Mitarbeitererzählungen an. Eine ausführliche Beschreibung in Anwendung auf den Unternehmenskontext gibt Beyer (2018: 30 ff.) in seinem Buch StoryThinking.

4 ,Die großen Erzählungen der Moderne, etwa das von der Aufklärung etablierte Narrativ von der fortschreitenden Emanzipation der Vernunft oder das marxistische Narrativ der Befreiung des Menschen aus der Sklaverei der Lohnarbeit [...]“ (Zifonun 2019: 1). 
- ,Erzählsituation“ dient der „Bezeichnung einer Kommunikationssituation, in der erzählt wird“" (ebd.).

Dabei verweist die Autorin darauf, dass sie den Terminus ,Erzählsituation“ anders als im literaturwissenschaftlichen Sinne verwendet. In den Literaturwissenschaften geht die Bezeichnung ,Erzählsituation“ auf Stanzel zurück, der von drei typischen Formen ausgeht: der auktorialen Erzählsituation, der IchErzählsituation und der personalen Erzählsituation. Alle drei Typen beziehen sich, ohne das hier weiter vertiefen zu wollen, anders als die Begriffsverwendung von Gülich auf eine Art des Vermittlungsvorgangs ${ }^{5}$ und nicht auf die Situation. In den Literaturwissenschaften wird der Erzählakt nach Genette als narration, der Erzähldiskurs als discours und die Geschichte als histoire bezeichnet. Dabei ist es möglich, die beiden ersten Ebenen (narration und discours) gemeinsam als Erzählbericht unter dem Begriff discours zu verorten. (Fludernik 2013: 10 f.) Hinsichtlich des discours ist zu bedenken, dass die Literaturwissenschaften auf literarische Erzähltexte spezialisiert sind. Diese zeichnen sich im Gegensatz zu Alltagserzählungen dadurch aus, dass sie über vier Interaktionsebenen verfügen, zu denen jeweils bestimmte Sender/innen und Empfänger/innen gehören. Die Ebenen entstehen dadurch, dass man zwischen Autor-, Erzähler- und Figurenkommunikation unterscheidet: (1) die Ebene des/der konkreten Autor/in und Leser/in, (2) die Ebene des/der abstrakten Autor/in und Leser/in ${ }^{6}$, (3) die Ebene des/der fiktiven Erzähler/in ${ }^{7}$ und (4) die kommunikative Ebene zwischen den erzählten Figuren (vgl. Schwitalla/Tittula 2009).

Grundsätzlich stellt sich im Zusammenhang mit den Basisbegriffen rund um ,Narrativität ' die Frage: Was ist narrativ? Allerdings ist eine Antwort auf diese Frage schwerlich pauschal zu geben, da grundsätzlich das Untersuchungsinteresse und der Untersuchungsgegenstand die Antwort beeinflussen. Das Untersuchungsinteresse kann sich beispielsweise auf funktionelle oder strukturelle Aspekte der Narrativität richten. Dabei können strukturelle Aspekte dialogorientiert, kognitiv orientiert oder textstruktureller Natur sein. Der Untersuchungsgegenstand kann wiederum aus einem Kontinuum von privaten bis zu institutionellen Kontexten gewählt werden sowie mündlich, schriftlich oder filmisch repräsentiert sein.

\footnotetext{
${ }^{5}$ Zur Weiterentwicklung und Kritik an Stanzels Entwurf siehe Martinez/Scheffel (2007: 89 ff.).

${ }^{6}$ Sie entspr. der Vorstellung, die sich bei dem/der Rezipient/in während der Lektüre über den/die Autor/in entwickelt und umgekehrt.

${ }^{7}$ Sie betrifft fiktionale Erzählungen, in denen die Erzähler/in nicht mit dem/der Autor/in gleichgesetzt werden kann.
} 
Unter den verschiedenen Betrachtungsweisen entstehen unterschiedliche Merkmalsausprägungen, die nicht unbedingt verallgemeinerbar sind. Als Basis für die folgenden Kapitel leite ich nun mit dem Minimalkonsens ein, der in unterschiedlichen erzähltheoretischen Abhandlungen hinsichtlich des ,Narrativen“ besteht. Da die Eigenschaft ,narrativ` auf die Sprechhandlung ,Erzählen` und auf das daraus resultierende Ergebnis - die Geschichte - zurückgeht, kann man das Erzählerische über seine Voraussetzungen - also Merkmale des Erzählens und der Geschichte - fassen. Die folgende Ausführung ist auf nicht-literarische Texte gerichtet. Sie orientiert sich an einem interdisziplinär anwendbaren Ansatz von Martinez (2017), der auf die Frage eingeht: Was ist Erzählen? Ich nehme die geforderten Merkmale in textlinguistischen Zitaten auf, um die Gemeinsamkeiten zu verdeutlichen. Martinez (2017) wählt den Zugang zu der Frage über die Minimalbedingung „Erzählen ist Geschehensdarstellung“ (ebd. 2017: 2). Hierfür formuliert der Autor drei notwendige Merkmale (Konkretheit, Temporalität, Kontiguität, Martinez 2017: 2) und ergänzt die Minimalbedingung mit „, $+\mathrm{x}^{\text {“, das }}$ symbolisch für optionale Bedingungen steht. Dazu mehr im nächsten Kapitel. Zuerst geht es um die Minimalbedingung, Geschehensdarstellung .

Unter ,Darstellung " versteht der Autor eine „Bezugnahme auf ein Geschehen“ (ebd. 2017: 1), die unabhängig von einem vermittelnden Medium ist. Damit ist Martinez‘ Explikation losgelöst von der Mittelbarkeit, auf die für gewöhnlich in Form eines engen Narrationsverständnisses Bezug genommen wird. Ein enger Begriff von Narrativität ist an eine erzählerische Vermittlungsinstanz gebunden. Eine weite Definition setzt dagegen ,das Merkmal der erzählten Handlung“ (Nünning/Nünning 2002: 7) voraus, das über das Verbale hinausgeht. Ein Geschehen beruht auf einem oder mehreren Ereignissen. Das Ereignis als kleinste, ,elementare Einheit eines narrativen Textes" (Martinez/Scheffel 2007: 25, 108) findet sich ebenfalls in den exemplarisch ausgewählten, textlinguistischen Zitaten als Kriterium wieder:

- „aufeinander folgende Ereignisse bzw. Situationen“ (Labov/Waletzky 1973: 95)

- eine ,zeitlich geordnete Abfolge von Handlungen, die wir als Ereignis kennzeichnen wollen“ (Heinemann/Viehweger 1991: 238, Hervorhebung i.O.)

- eine „Darstellung vergangener Ereignisse“ (Kern 2000: 99)

- eine „Repräsentation des Ereignisses“ (Brinker et al. 2014: 66)

An das Ereignis werden wiederum unterschiedliche Kriterien geknüpft, die jeweils spezifisch für das Untersuchungsobjekt sind. Mit dem Ereignis eng 
verbunden ist nach Martinez (2017) das erste obligatorische Merkmal der Geschehensdarstellung: das Merkmale der Konkretheit. Darunter versteht er, dass eine Erzählung ,singuläre und konkrete Gegenstände und Sachverhalte [wiedergibt]“ (ebd. 2017: 1). Ähnlich formulieren Brinker et al. (2014) die Anforderung mit der Aussagen: „Das Thema einer Erzählung wird durch ein abgeschlossenes, singuläres Ereignis repräsentiert" (Brinker et al. 2014: 66). Für die Darstellung des Geschehens spielt die Verknüpfung der Geschehnisse (Zifonun 2017: 1 f.) eine grundlegende Rolle. Sie ergibt sich durch eine ,zeitliche und räumliche Abfolge von Handlungsschritten, Ereignissen und Erlebnissen“ (Tophinke 2017: 71). Diese Charakterisierung steht in engem Bezug zu den zwei letzten obligatorischen Merkmalen der Minimalbedingungen nach Martinez. Das betrifft die Merkmale Temporalität und Kontiguität. Die Temporalität ist in Martinez' Betrachtung für das Erzählen funktional, da sie eine Geschehensdarstellung entlang eines Zeitverlaufes gliedert: Mithilfe von Temporalität ordnen Erzähler/innen das Geschehen in einen chronologisch geordneten Ablauf, der das Geschehen in einem ,vorher vs. nachher“ (Martinez 2017: 2) verdeutlichen. Ist eine Erzählung ,zu einem kohärenten Ganzen“ (Saupe/Wiedemann 2015: 4) geformt, erfüllt sie das Merkmal der Kontiguität nach Martinez. Der Autor versteht darunter, dass zwischen den Ereignissen der Geschehensdarstellung zumindest ,implizit eine kausale Verbindung" (Martinez 2017: 3) besteht. Das bedeutet, die Ordnung der Ereignisse in ein Vorher und ein Nachher (= chronologische Abfolge) muss darum ergänzt werden, dass die Ereignisse räumlich, zeitlich oder kausal aufeinander bezogen sind. In der mündlichen Erzählforschung kommt hinzu, dass Erzählen als eine rekonstruktive und kommunikative Tätigkeit (vgl. Gülich/Hausendorf 2000: 369; Quasthoff 1980: 12) verstanden wird. Dabei sind vor allem funktionelle Aspekte von Interesse, die aufgrund der vielfältigen Untersuchungsobjekte zahlreich sind. Quasthoff (1980) unterscheidet daher Funktionen, die auf die Erzähltextinhalte abzielen und solche, die mit der Erzählaktivität verbunden sind. Dabei unterscheidet sie zwischen inhaltsbasierten, formbasierten und interaktiven Funktionen. (Hausendorf/Quasthoff 1996: 12) Solche Funktionen sind mit Blick auf diese Arbeit beispielsweise:

- Selbstdarstellung und Identitätskonstruktion (Quasthoff 1980, 1996; LuciusHoene/Deppermann 2004; Krüger 2015; Burel 2015; Günthner 2012; Kern 2000; Tophinke 2017 u. a.)

- Wirklichkeitskonstruktion (Mell/Gredel 2018; Klein/Martinez 2009; Kern/Morek/Ohlhus 2012 u. a.) 
- Unterhaltung (Quasthoff 1980: 159; Klatschgeschichten: Bergmann 1987; Kotthoff 2017)

- Bedeutungs-/Sinnstiftung (Mell/Gredel 2018; Lucius-Hoene/Scheidt 2017)

Zusammenfassend lässt sich, ausgehend von Martinez' Minimalbedingungen für das Erzählen, festhalten, dass etwas narrativ ist, wenn es ein Geschehen darstellt. Das Geschehen besteht aus mindestens einem Ereignis, das sich als Erzählgegenstand qualifiziert. Dabei strukturiert eine temporale Verknüpfung die Geschehensdarstellung. Die erwirkte Abfolge von Handlungen und Ereignissen steht darüber hinaus in einem kohäsiven Verhältnis zueinander. Das bedeutet, dass sie nicht nur zeitlich geordnet, sondern auch zeitlich, räumlich und kausal verbunden sind. Zusätzlich adaptiere ich die zwei linguistischen Merkmale, nach denen Erzählen eine rekonstruktive und kommunikative Tätigkeit ist, die vorwiegend funktional für eine imagefördernde (S)D ist. Im Folgenden geht es ergänzend um spezifische narrative Merkmale, die bei Martinez (2017) unter den Platzhalter " $+x^{\prime \prime}$ fallen. Sie sind optional und können daher variabel je nach Untersuchungsgegenstand angewandt werden. Zugleich verdeutlicht das folgende Kapitel narrative Kennzeichen, die für die erhobenen uRE bezeichnend sind.

\subsection{Gegenstandsbezogene inhaltliche Merkmale: das optionale " $+\mathrm{x}^{\prime \prime}$}

Martinez (2017) beantwortet die Frage Was ist Erzählen? mit der Formel ,Geschehensdarstellung $+x^{c}$. Der Platzhalter,$+x^{6}$ steht für verschiedene Vorschläge von Narratolog/innen, die beispielsweise auf die Beschaffenheit des Ereignisses oder des Geschehens abheben. Die Merkmale sind objektspezifisch. Erkennbar ist das daran, dass sich für ein festgelegtes Merkmal in anderen Bereichen narrative Beispiele finden lassen, die das Merkmal wiederum ausschließen (Martinez 2017: 3).

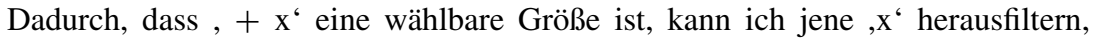
die der jeweiligen uRE entsprechen und sie darüber zugleich aus der narratologischen Perspektive als Untersuchungsgegenstand qualifizieren. Zusätzlich ermöglicht diese Perspektive das heterogene Korpus narratologisch zu erfassen. Aus der Vielzahl dieser linguistisch und literaturwissenschaftlich formulierten Kriterien habe ich eine dementsprechende Auswahl getroffen. Grundsätzlich zielen die Merkmale darauf, dass sie die Geschehnisse erzählenswert erscheinen lassen. Die Betrachtungsweise geht auf Labov (1972) zurück: 
,[I]t is necessary to know why this narrative - or any narrative - is felt to be tellable;

[...]" (Labov 1972: 370).

Die Zuhörer/in soll durch entsprechende Merkmale der Geschehensdarbietung nicht nach dem „So what?“ (Labov 1972: 366) - also dem Sinn der Erzählung - fragen müssen. Die damit verbundene Erzählwürdigkeit bindet der Autor an die Evaluation zurück, die er als ,perhaps the most important element in addition to the basic narrative clause" (ebd.) gewichtet. Arbeiten aus der neueren linguistischen Forschung beziehen sich regelmäßig auf die Erzählwürdigkeit, die Erzähler/innen auch mit anderen Mitteln wie der Evaluation ausdrücken können. Quasthoff (1980: 52) verwendet unter Berufung auf die ,reportability (= Erzählwürdigkeit) das Adjektiv ,erzählenswert‘. Der Ausdruck verdeutlicht m. E. aufgrund der zweiten Konstituente, wert', dass mit Erzählungen funktionale Erwägungen einhergehen, die es einzulösen gilt. Eine Erzählung als erzählenswert auszuweisen, ist für diese Arbeit aufgrund des Kontextes äußerst bedeutend. Mit einer erzählenswerten Geschichte ist jene „Attraktivität“ verbunden, die für eine Aufmerksamkeitsweckung sowie -bindung und für die Verbreitung der imagefördernden (S)D maßgeblich ist.

Die von mir ausgewählten optionalen Merkmale sind auf die Geschichte gerichtet, denn das Erzählenswerte liegt ,zu einem ganz wesentlichen Teil bereits in der Geschichte" (Quasthoff 1980: 52). Hierbei beziehe ich mich auf zwei linguistische Perspektiven, die auf das aufgefundene narrative Vermittlungsvorkommen in hohem Maße zutreffen. Da die erhobenen uRE durch den institutionellen Kontext keine natürlichen Erzählungen sind und die Aufbereitung der Geschichte so unterschiedlich wie die Erzähler/innen (professionalisierte Autorenkollektive bzw. Erzähler/in mit „Erzählkenntnissen“ vs. ,intuitive“ Erzähler/in) ist, gehe ich im Anschluss daran auf zwei grundlegende Betrachtungsweisen aus den Literaturwissenschaften ein, die die uRE ebenfalls kennzeichnen.

\subsubsection{Spezifizierende Merkmale aus der linguistischen Erzählforschung}

Da der Ausgangspunkt der vorliegenden Arbeit Erzähltexte sind, leite ich die Merkmale aus dem Erzählprodukt ab und nicht aus der Rezeption. In der vorgenommen Auseinandersetzung damit, was eine uRE als erzählenswert ausweist, geht es um Merkmale, die auf die Erlebnisqualität des erzählten Geschehens rückschließen lassen. Für die erhobenen uRE sind aus den möglichen Merkmalen zur Etablierung des Erzählenswerten das Kriterium der Bewertung von 
Labov/Waletzky $(1967 ; 1973)$ und zum anderen das sozial relevante außergewöhnliche Ereignis nach Kindt (1997) bezeichnend. Beide Ansätze stammen zwar aus der mündlichen Erzählforschung, doch herrscht in der Narratologie Konsens darüber, dass Bewertungen für Erzählungen kennzeichnend sind. Das von Kindt erwogene Kriterium entstand in der Auseinandersetzung damit, was für eine adäquate Erzähldefinition erforderlich ist. Hierfür bezieht sich der Autor ebenfalls auf ,dialogische Texte der mündlichen nichtliterarischen Kommunikation“ (Kindt 1997: 2). Doch auch in diesem Fall kann man nicht behaupten, das Merkmal sei an die Mündlichkeit gebunden.

Labov/Waletzky untersuchten 1967 ,prototypische Strukturen von elizitierten, mündlichen Erzählungen“ (Kotthoff 2017: 4). Als Datengrundlage dienten ihnen sechshundert Tonbandinterviews von Sprecher/innen, die über keine hohe Schulbildung verfügten (ebd.: 80 f.). Ihr Ziel war es, ,die formalen Eigenschaften der Erzählung mit ihren Funktionen in Beziehung zu setzen“ (Labov/Waletzky 1973: 78). Die beiden Forscher stellten fest, dass die untersuchten Erzählungen zwar weitgehend verschiedene Oberflächenstrukturen aufweisen, diese aber über äquivalente semantische Interpretationen verfügen (Labov/Waletzky 1973: 108). Labov/Waletzky erhoben eine Grundform für Erzählungen (= Primärfolge, ebd.: 110), die formal ,auf dem Kriterium der Verschiebbarkeit von Sätzen aufbau[t]“ (Quasthoff 1980: 32). Die daraus entnommene Oberflächenstruktur zeigt sich in einer linearen Ereignisabfolge der erzählten Handlungen mit den Elementen: Orientierung (Angaben zu Ort, Zeit, Personen und Handlungssituation), Komplikation (Ereignisabfolge mit einem außergewöhnlichen Ereignis), Auflösung (der Komplikation) und ggf. Coda (Rückbindung der Erzählung in das Hier und Jetzt). Mittels der Evaluation wird letztlich die Primärfolge in die Normalform der Erzählung transformiert. (vgl. ebd.) Diese formalen Eigenschaften einer Erzählung setzten die Autoren mit ihrer sprachlichen Funktion in Beziehung. Diesbezüglich erhoben sie eine referenzielle und eine evaluative Funktion von Sprache. Während sich die referenzielle Funktion auf eine lineare Äußerungsabfolge konzentriert, bezieht sich die evaluative Funktion auf „die Einstellung des Erzählers gegenüber seiner Erzählung“ (Labov/Waletzky 1973: 119), also der Bewertung des Erzählten. Diese entspricht dem Element der Evaluation. Ist die Evaluation in der mündlichen Kommunikation an den/die Erzähler/in gebunden, kann sie der/die Erzähler/in in einem schriftlichen Erzähltext auch auf zitierte Personen übertragen, als eine Art „Stellvertreter-Bewertung“. Dadurch sind für die Analyse neben Evaluationen der Erzähler/innen auch evaluative Äußerungen zitierter Personen von Interesse. Ursprünglich verorteten Labov/Waletzky die Evaluation in der festen Stellung zwischen der Komplikation und der Auflösung, was sie später aufgrund empirischer Belege (siehe z. B. Quasthoff 1980: 32 f.; 
Gülich 1976: 252) als flexibel modifizierten. Dafür, inwiefern eine Erzählung als erzählenswert erscheint, spielt die evaluative Funktion eine zentrale Rolle. Die Evaluation, so Labov/Waletzky, zeigt die „Wichtigkeit bestimmter narrativer Einheiten“ (ebd. 1973: 120) und hebt sie gegenüber anderen Einheiten hervor. Einer Erzählung ohne Evaluation „geht Signifikanz ab: sie hat kein Ziel“ (Labov/Waletzky 1973: 114). Dass die Evaluation die Einstellung des/der Erzähler/in zu ihrer Geschichte anzeigt, legt auch nahe, dass er/sie darüber ihre Erzählung als erzählenswert ausweist. Allerdings sind Evaluationen nicht nur darauf beschränkt, ,inwiefern die Geschichte für den Kommunikations- bzw. Interaktionszusammenhang relevante [ist]“" (Gülich 1976: 252). In Gülichs Korpus liegen solche Evaluationen weniger im Erzähltext, sondern eher als ausleitende Bemerkung am Ende vor (ebd.). Um die Eigenschaft ,erzählenswert zu sein` zu markieren, ist vor allem die subjektive Bedeutung des Ereignisses und seine Erlebnisqualität für die Erzähler/innen von Interesse. Damit ist wiederum eine emotionale Komponente verbunden, worüber Evaluation und Emotion eng miteinander verbunden sind (vgl. Gülich/Hausendorf 2000: 374). Labov/Waletzky verorten anhand ihrer Definition die Evaluation unter der Kategorie ,Funktion". Quasthoff merkt hierzu kritisch an, dass diese Grenzziehung der Autoren zwischen der Kategorie ,Funktion" und der Kategorie ,Struktur' in Bezug auf die Evaluation vage ist ${ }^{8}$. Zwar betrachten Labov/Waletzky die Evaluation als funktionelle Kategorie, doch implizit hat sie aufgrund der strukturellen Bestimmung auch eine besondere strukturelle Bedeutung. (Quasthoff 1980: 34) Quasthoff betrachtet daher evaluative Elemente als ,formale Mittel, um die intendierte Funktion wirksam zu machen“ (ebd., Hervorhebung i.O.), also als Darstellungsmittel. Um die Frage zu lösen, ob die Evaluation nun eher der Kategorie ,Funktion' oder der Kategorie ,Struktur' zuzurechnen ist, unterscheidet Quasthoff zwischen $G$ - und E-Evaluationen. G-Evaluationen beziehen sich auf Teile der Geschichte. Dadurch sind sie „Formen der semantischen Repräsentation“ (Quasthoff 1980: 38). EEvaluationen beziehen sich dagegen ,nicht auf Teile der erzählten Geschichte zum Zeitpunkt des Ablaufs der Ereignisse, sondern auf Teile der Erzählung zum Zeitpunkt des Erzählens“ (ebd., Hervorhebung i. O.). Sie erfüllen auf dieser Ebene eine textstrukturierende Funktion. Aus diesem Grund sind hier als inhaltliches Differenzkriterium vor allem G-Evaluationen von Interesse. Sie stellen

\footnotetext{
${ }^{8}$ Zur Vermischung von Definitionsbedingungen und Hypothesen auch Kindt (1997: 3): ,So bleibt nämlich unklar, inwieweit die Existenz von Komplikation und Evaluation in Erzählungen den Status von Definitionsbedingungen oder Strukturhypothesen haben sollen.“
} 
einen Anknüpfungspunkt für die emotionale, subjektive Bedeutung und Erlebnisqualität seitens der Erzähler/innen dar. Hinsichtlich evaluativ sprachlicher Mittel unterscheiden Labov/Waletzky:

- semantisch definierte Mittel: direkte Feststellung / lexikalische Intensifikatoren

- formal definierte Mittel: Wiederholungen

- kulturell definierte Mittel: symbolische Handlung (z. B. sich bekreuzigen), das Urteil einer dritten Person (Labov/Waletzky 1973: 120)

Diese sprachlich evaluierenden Mittel finden sich ebenfalls in den Daten: so zum Beispiel affektiv assertive Sprechhandlungen, Intensivierer oder Wirklichkeitsmarker als semantisch definierte Mittel, Reformulierungen und Interpretationen von wörtlichen Zitaten als formal definierte Mittel. Unabhängig davon, ob die Evaluation semantisch oder textstrukturell ausgerichtet ist, sind Evaluationen für die (S)D von zentraler Bedeutung. Ursächlich dafür ist, dass die Erzähltextinhalte sich im Normalfall auf die Unternehmen beziehen. So richten sich die Beurteilungen mal mehr, mal weniger deutlich ebenfalls auf die Unternehmen. Daher ist es nicht verwunderlich, dass in den uRE häufig Evaluationen auftreten. Allerdings sind die Erzähler/innen in Bezug auf ihre Beurteilung nicht vollkommen frei. Allzu schnell kann ein Übermaß oder eine zu positive Bewertung sich in eine ungünstige (S)D verkehren. In diesem Zusammenhang zeigt sich, je expliziter eine positive Evaluation ausfällt, desto stärker wird sie auf das persönliche Erleben und die Erlebnisqualität von Erzähler/innen (für gewöhnlich Mitarbeiter/innen) oder externer Personen ausgelagert. Das betrifft beispielsweise uRE über öffentlichkeitswirksame Maßnahmen, die unter die Stilisierung des Grundtyp 3 (das verantwortungsbewusste Unternehmen, Abschn. 8.5) fallen. Nimmt ein/e Erzähler/in die Bewertung selbst vor, schützt er/sie mit einer persönlichen emotionalen Betroffenheit seine/ihrer Evaluation davor, dass seine/ihre Bewertung in Frage gestellt werden kann. Emotion und Evaluation sind in diesem Kontext besonders eng aneinander gebunden. In Bezug auf die emotionale Qualität einer Erzählung, hält auch Kindt (1997: 19) fest, dass ein erzählerisch dargestelltes Geschehen, welches mit starken Emotionen für die Beteiligten einhergeht, die Erzählwürdigkeit deutlich anhebt. So formuliert er:

„Eine prototypische Erzählung enthält zu einem wesentlichen Anteil Äußerungsformen, aus denen eine Zuordnung emotiver Bedeutungen für das dargestellte Geschehen und seine Konsequenzen resultieren.“ (Kindt 1997: 19) 
Das zweite Merkmal, auf das ich mich hinsichtlich der uRE beziehe, lässt sich mithilfe der vierten von insgesamt fünf Definitionsbedingungen des Erzählens nach Kindt (1997) erfassen. Das Ziel des Autors ist es, das Erzählen mithilfe der Definitionsbedingungen von anderen Kommunikationsmodi - insbesondere dem Berichten - abzugrenzen. Das Merkmale, auf das ich im Folgenden referiere, lautet:

„(D4) Das in einer prototypischen Erzählung dargestellte Geschehen enthält mindestens ein sozial relevantes außergewöhnliches Ereignis.“ (Kindt 1997: 18)

Voraus geht der Bedingung die Auseinandersetzung damit, was ein Geschehen ausmacht, nämlich:

„(3) Eine prototypische Erzählung besteht zum überwiegenden Teil aus der Darstellung eines Geschehens [...].“ (Kindt 1997: 12)

Das Geschehen spezifiziert der Autor, indem er es als eine „temporal geordnete, zusammenhängende Folge von Zuständen eines dynamischen Systems [...], die auch Prozessanteile enthält" (Kindt 1997: 13), beschreibt. Dabei stellen Ereignisse, ,die prototypisch durch einmalige, momentan erfolgende, ausgeprägte Zustandswechsel ausgezeichnet sind" (ebd.: 13), einen Spezialfall von Prozessen dar. Um von dieser Basis ausgehend das Ereignis im Sinne einer Erzählung weiter zu spezifizieren, präzisiert er die Definitionsbedingung (4) in Anlehnung von Labov's/Waletzky's reportability. Die Problematik des Konzepts der ,reportability" sieht der Autor darin, dass es sich um eine Teilnehmerbeurteilung handelt, die er nicht auf der Ebene der Geschehensbeschreibung sieht. Eine prototypische Erzählung solle mithilfe eines überindividuellen konkreten Kriteriums charakterisiert werden. Zur Veranschaulichung geht Kindt auf das Kriterium von Quasthoff (1980) - den Planbruch - und auf den Diskontinuitätsbegriff von Boueke et al. (1995) ein. Ein Planbruch ist eine „unerwartete Wendung im Geschehen“ (Hausendorf/Quasthoff 1996: 84), die darauf beruht, dass das Planziel eines Akteurs zunichte gemacht oder in Frage gestellt wird (Quasthoff 1980: 49). Kindt sieht das Kriterium des Planbruchs, der einen Spezialfall der ,reportability nach Labov/Waletzky darstelle, insofern als problematisch an, da es unzweckmäßig sei, ,die Wahrnehmung eines subjektiv erwarteten Ereignisses als ,Beobachterplanbruch“ zu rekonstruieren“ (Kindt 1997: 15). Aufgrund dessen erachtet Kindt die Bedingungsexplikation als unzureichend. Ein ähnliches Problem sieht er bei dem Diskontinuitätsbegriff von Boueke et al. (1995: 83), der sich auf die postulierte Bedingung von van Dijk (1977) stützt, dass das zentrale Ereignis in einer 
Erzählung nicht erwartbar, also unerwartet, sei. Ähnlich wie im Fall des Planbruchs sieht Kindt die Erwartbarkeit eines Ereignisses der Teilnehmerbewertung zugehörig. Geeigneter findet er die ebenfalls auf van Dijk (1977) zurückgehende Formulierung, ,dass eine Ausnahme vom normalen Ereignisablauf und in diesem Sinne eine Außergewöhnlichkeit vorliegt" (Kindt 1997: 16).

Das Außergewöhnliche erfasst Kindt als eine Abweichung von der Normfallregularität. Die Normfallregularität basiert auf Verhaltensregularitäten von Systemen (z. B. kognitive Schemata), die es uns möglich machen, Verhalten zu erklären und/oder zu prognostizieren. In der Kommunikation und Interaktion zeige sich, dass Menschen ,in starkem Maße von Regularitäten Gebrauch machen, die das Verhalten eines Systems 'im Normalfall' charakterisieren" (Kindt 1997: 17). In vielen Fällen haben Vorgangsbeschreibungen die Funktion, einen Normalfall darzustellen. Zur Lebensbewältigung ist das jedoch unzureichend, denn man muss auch mit Abweichungen von den Regularitäten rechnen „und diese vermeiden oder darauf vorbereitet sein, um angemessen handeln zu können“ (ebd.). Darin sieht Kindt „eine zentrale kognitive Funktion“ (Kindt 1997: 17) von Erzählungen. Vor dem beschriebenen Hintergrund konstituiert sich die Außergewöhnlichkeit dadurch, „dass mindestens ein Ereignis von dem durch Normalfallregularitäten charakterisierbaren Standardverhalten des betrachteten Systems abweicht“ (ebd.). Die besondere Relevanz erhält eine solche Abweichung aufgrund der positiven oder negativen Konsequenzen, die sie für die Beteiligten der Interaktionsgemeinschaft hat. Sozial relevant wird das Ereignis dann, wenn bestimmte Personen von den Konsequenzen konkret betroffen sind. Die Ausrichtung des Außergewöhnlichen an der Normfallregularität verdeutlicht zugleich, dass die Einschätzung darüber, ob ein Ereignis außergewöhnlich ist, zeitabhängig und kontextabhängig ist: Je nachdem für welchen Kontext die Normalfallregularität angesetzt ist. Der Vorteil dieser Betrachtungsweise für das vorliegende Projekt besteht in zwei Punkten. Erstens ist das Kriterium ,Außergewöhnlichkeit" durch die Charakterisierung, sozial relevant" an der Betroffenheit der Akteure erkennbar, die sprachlich nachvollziehbar ist (z. B. Distanzierung, Begeisterung, Identifikation u. a.). Zum anderen muss das Außergewöhnliche nicht unbedingt einen Höhepunkt (z. B. in Form einer Komplikation) beinhalten, da das nur ein Ausdruck von sozialer Relevanz ist. Die Daten zeigen, dass sich nicht in allen uRE ein „klassischer“ Höhepunkt oder ein Wendepunkt abzeichnet. Das Ereignis oder das Geschehen wird stattdessen als besonders sozial relevant für bestimmte Akteure ausgezeichnet. Das spiegelt sich wiederum in dem Merkmal der Erfahrungshaftigkeit (Fludernik 1996). 


\subsection{2 Übernahme literaturwissenschaftlicher Merkmale}

Aus der Literaturwissenschaft sind inhaltliche Merkmale aus zwei Modellen für die untersuchten uRE von Interesse. Nach wie vor geht es in diesem Unterkapitel um inhaltliche Merkmale, die eine Erzählung als erzählenswert auszeichnen. Daher werden nicht die vollständigen Ansätze, sondern die dementsprechenden Merkmale aufgegriffen. Das ist zum einen das Merkmal der Erfahrungshaftigkeit, das Fludernik in ihrem natürlichkeitstheoretischen Ansatz (,Natural Narratology', Fludernik 1996) als Kernelement von Erzählungen fasst. Sie versteht das Merkmal als obligatorisch und nicht als optional, wie es in dem vorliegenden Kontext angewandt wird. Zum anderen handelt es sich um das narrative Elemente der Zustandsveränderung (Schmid 2014: 3), das Schmid u. a. als Minimalbedingung für Narrativität ansieht. Auch hier ist das Merkmal für den Autor obligatorisch. Für den vorliegenden Kontext nehme ich die Merkmale jedoch als optional auf, damit sie je nach Vermittlungsvorkommen anwendbar sind.

Fludernik kombiniert in ihrem Modell der Natural Narratology Erkenntnisse der Literaturtheorie mit denen der Linguistik. Die Autorin bezieht in ihr Modell kognitive Aspekte ein, die sie mit dem Begriff ,natural' insofern einschränkt, als sie sich ausschließlich auf kognitive Rahmen (Frames) beziehen, auf deren Grundlage Texte interpretiert werden (Fludernik 1996: 12). Sie verbindet den Bereich mit dem soziolinguistischen, indem sie sich auf natürliche Erzählungen (natural narratives) bezieht. Darunter versteht sie

\footnotetext{
„mainly, spontaneous conversational storytelling, [...]. Natural narrative, in contradistinction to the wider area of oral narrative, comprises only spontaneous forms of (therefore conversational) storytelling but excludes oral poetry and folktale traditions of oral storytelling." (ebd. 1996: 13 f.)
}

Dabei geht Fludernik davon aus, dass bestimmte kognitive Ankerpunkte existieren, die eine Erzählung konstituieren. Die Ankerpunkte sieht sie in der emotionalen Beteiligung an der Erfahrung und ihrer Bewertung. Sie argumentiert, dass Erfahrungen als emotional aufgeladene Erinnerung gespeichert und in narrativer Form reproduziert werden (Fludernik 1996: 29). Dadurch entsteht eine emotionale Involviertheit der Erzähler/in, die die Bedeutsamkeit der erzählten Erfahrung greifbar macht und sie darüber als erzählenswert ausweist. Die Darstellung der subjektiven Erfahrung von Wirklichkeit durch eine/n Ich-Erzähler/in oder Figuren in einer fiktionalen Erzählung (vgl. Martinez 2017: 5) nennt Fludernik die Erfahrungshaftigkeit (Experientiality): „the quasi-mimetic evocation 
of real-life experience“ (ebd. 1996: 12). Dieses Merkmal führt dazu, dass die Geschehensdarstellung an sich kein notwendiges Merkmal mehr darstellt:

"In my model there can therefore be narratives without plot, but there cannot be any narratives without a human (anthropomorphic) experiencer of some sort at some narrative level." (Fludernik 1996: 13)

Dadurch ist jeder Text, der Erfahrung darstellt, für Fludernik ein narrativer Text. Unabhängig davon, ob er eine eindeutige Folge von kausal zusammenhängenden Ereignissen und Handlungen abbildet (vgl. Caracciolo 2014: 2), da „Erzählen grundsätzlich an eine menschliche Perspektive des Erlebens gebunden ist" (Rinke 2012: 298). Nicht alle Narratolog/inen stimmen mit dieser Grundannahme überein. Beispielsweise stimmt Martinez dem Ansatz zwar zu, ,dass die Repräsentation von subjektiver Erfahrung zweifellos ein wichtiger Grund dafür ist, weshalb wir uns überhaupt für Erzählungen interessieren“ (Martinez 2017: 5), erachtet das Merkmal allerdings nicht als notwendig oder hinreichend um ausschließlich darauf Narrativität zu begründen. Er argumentiert, dass es sowohl Texte gibt, die als narrativ erachtet werden, obwohl sie keine subjektiven Erfahrungen als Merkmale von Erfahrungshaftigkeit tragen (z. B. Erzählungen im neutral beobachtenden Stil). Ebenso existierten Texte, die zwar persönliche Erfahrungen beinhalten, jedoch nicht als narrativ aufgefasst werden (z. B. Naturgedichte). (Martinez 2017: 5) Dadurch komme dem Merkmal keine diskriminierend Eigenschaft zu. Darüber hinaus merkt Caraccido (2014: 1) kritisch an, dass in dem Modell unklar bleibe, ob die Erfahrungshaftigkeit als eine textinhärente Eigenschaft angesehen wird, die inhaltlich identifizierbar ist, oder ob sie darin besteht, dass sie in der Text-Rezipienten-Interaktion als psychologischer Prozess ausgelöst wird. Für die vorliegende Arbeit verorte ich die Erfahrungshaftigkeit als ein inhaltliches Merkmal, das textimmanent ist und über die Sprachverwendung ersichtlich wird. Hierbei ist das Merkmal insbesondere in Form des subjektiv bewertenden Ausdrucks erkennbar, das sich vornehmlich in uRE über den Arbeitsplatz unter dem Einfluss des employer branding zeigt. Deutlich wird das anhand der sprachlichen Vermittlung von starken positiven Emotionen wie Glück und Freude über und an dem Arbeitsplatz sowie Überraschung über Unternehmensangebote und eine wohlwollende familiäre Unternehmensgemeinschaft (z. B. für die Stilisierung des Grundtyp 4 zur (S)D als attraktiver Arbeitgeber, Abschn. 8.6). Darüber hinaus handelt es sich um ein strategisches Moment, denn durch persönliche Erfahrungen gewinnt die uRE an Lebendigkeit. Zum anderen präsentieren die Erzähler/innen ihre Sicht auf die Unternehmenswelt, die die Rezipient/innen durch die Augen 
der Betroffenen erleben und bestenfalls übernehmen sollen. Doch nicht jedes narrative Vermittlungsvorkommen in dem Korpus weist einen so hohen Grad an Erfahrungshaftigkeit auf. Insbesondere Erzählungen, die nicht von einem $/ \mathrm{r}$ Ich-Erzähler/in verfasst sind, können kein erlebendes Ich vorweisen, sondern „nur“ das erzählende Ich (heterodiegetische Erzählung, vgl. Martinez/Scheffel 2007: 81). Einen Teil der Daten kennzeichnet, dass die Erzähler/in ihre uRE anhand von Informationen Dritter konstruiert. Dabei üben die Erzähler/innen äußerste Zurückhaltung in Bezug auf ihre Person. Das bedeutet, sie lagern Bewertungen allenfalls in Zitate aus und vermeiden es, die Außerordentlichkeit des Ereignisses zu kommunizieren. Aufgrund der narrativen Darbietung ist das Merkmal der Experientiality auf diese uRE nicht anwendbar. Dennoch handelt es sich hierbei um Erzählungen.

Inwiefern sich diese Erzählungen als erzählenswert ausweisen, lässt sich mithilfe des Merkmals der Zustandsveränderung (Schmid 2014) erfassen. Das Merkmal resultiert aus der erzählten Handlung. Dadurch gehört es ebenfalls zu den inhaltlichen Merkmalen einer Erzählung. Es entstammt einer strukturalistischen Perspektive. Konzepte mit diesem Hintergrund zeichnen sich dadurch aus, dass sie sich auf das Erzählte konzentrieren und nicht auf Merkmale des Diskurses. Im Mittelpunkt des Interesses steht ,ein bestimmte[r] Aufbau des Dargestellten“ (Schmid 2014: 2). Im strukturalistischen Sinne unterscheiden sich Erzählungen von Beschreibungen, da sie eine temporale Struktur aufweisen und eine Zustandsveränderung darstellen. Während klassische Konzepte das Erzählen an die Vermittlung binden, somit also eine vermittelnde Erzählinstanz vorhanden sein muss (vgl. enge Erzähldefinition), lösen strukturalistische Konzeptionen sich davon. So kann Narrativität durch jegliches Medium - also nicht ausschließlich durch Verbalität - vermittelt werden. Allerdings sind alle Darstellungen ausgeschlossen, ,deren Referent keine temporale Struktur besitzt und deshalb keine Veränderung enthält" (Schmid 2014: 2). Ein Zustand wird danach unterschieden, ob er sich auf den inneren Zustand einer Figur bezieht oder auf die äußere Welt. In Bezug auf die uRE bedeutet das, dass die Unternehmen als Agens die Zustandsänderung in ihren Geschichten verursachen. Dabei gestalten die Verfasser/innen die Erzähltextinhalte so, dass das Unternehmen eine positive Zustandsveränderung bewirkt. Unter einem Zustand wird

„eine Menge von Eigenschaften [verstanden], die sich auf eine Figur oder die Welt in einer bestimmten Zeit der erzählten Geschichte beziehen." (Schmid 2014: 3)

Daraus folgt, dass die positive Zustandsveränderung sich auf die innergeschichtlichen Akteure sowie die erzählte Welt beziehen kann. Damit etwas als Erzählung 
deklariert werden kann, ist die Voraussetzung, ,dass mindestens eine Veränderung eines Zustands in einem gegebenen zeitlichen Moment dargestellt wird" (ebd.: 3). Hierfür stellt Schmid drei Bedingungen an eine Zustandsveränderung, damit sie für eine Erzählung konstitutiv ist:

- „Eine temporale Struktur mit mindestens zwei Zuständen, einem Ausgangsund einem Endzustand [...].

- Eine Äquivalenz von Ausgangs- und Endzustand, d. h. Similarität und Kontrast der Zustände [...].

- Die beiden Zustände und die sich zwischen ihnen ereignende Veränderung müssen sich auf ein und dasselbe Subjekt des Handelns oder Erleidens oder auf ein und dasselbe Element des „setting“ beziehen [...].“ (Schmid 2014: 3 f., Hervorhebung i. O.)

Das Merkmal der Zustandsveränderung ist insbesondere für Erzählungen fruchtbar, in denen die Merkmale ,Erfahrungshaftigkeit" und ,sozial relevante Außergewöhnlichkeit' sowie ,Bewertungen' nicht funktionieren. Das betrifft beispielsweise Unternehmensgeschichten. In ihnen steht das Unternehmen als anthropomorpher Akteur im Mittelpunkt, dem jedoch im Sinne des Wirklichkeitsbezugs nicht ernsthaft affektive Regungen zugesprochen werden können (z. B. Abschn. 7.2.2.2). Ebenso werden Bewertungen implizit durch „objektivierende“ Maßnahmen wie quantitative Vorher-Nachher-Vergleiche oder die Anordnung der Ereignisse erzielt, jedoch nicht expliziert oder gar emotionalisiert. Dennoch erfüllen solche Erzählungen die erforderlichen Bedingungen. In der Geschichte wird ein Anfangs- und ein Endzustand (z. B. in Zeiten der Krise) in seinem zeitlichen Rahmen präsentiert. Similarität besteht darin, dass es sich um den Zustand des Unternehmens handelt, der durch den Anfangs- und Endzustand kontrastiert wird. Als handelndes Subjekt tritt das anthropomorphe Unternehmen auf, das entsprechende Entbehrungen oder Einbußen erleidet. Dabei ist nach Schmid das Kriterium für Narrativität hinreichend erfüllt, wenn die Veränderung durch die Darstellung von zwei miteinander kontrastierenden Zuständen impliziert ist (Schmid 2014: 3). Je nachdem kann die Zustandsveränderung so durchaus auch zu einem Merkmal werden, das die Geschichte als erzählenswert ausweist.

Das gewählte Vorgehen aus der bestehenden narrativen Grundlagenforschung theoretische Merkmale zusammenzustellen, dient dazu, Ankerpunkte zu erfassen, anhand derer sich die erhobenen uRE von nicht-erzählenden Texten abgrenzen. Die vier soeben beschriebenen Merkmale Bewertung, das sozial relevante Außergewöhnliche, die Erfahrungshaftigkeit und die Zustandsveränderung beziehen sich auf die Inhalte der Erzähltexte und vermitteln, inwiefern sie diese als 
erzählenswert ausweisen. Daneben existieren formale narrative Merkmale, die für Erzählungen charakteristisch sind. Von solchen Merkmale mit Bezug auf das Korpus handelt das folgende Unterkapitel. Das bedeutet, dieses Kapitel befasst sich nicht mehr mit Merkmalen des optionalen,$+x^{6}$.

\subsection{Gegenstandsbezogene formale Merkmale}

Gegenstandsbezogene formale Merkmale für Erzählungen rekurrieren auf textstrukturelle und sprachbezogene Aspekte aus narrativer Sicht. Aufgrund der unterschiedlichen Erhebungsorte und Realisierungsoptionen differieren die formalen Merkmale ebenso, wie zuvor die inhaltlichen. Berücksichtig werden muss dabei, dass Konzepte, die Erzähltexte als abgeschlossene und regelmäBige Geflechte formaler Konstanten begreifen, nur bedingt auf die strukturelle Beschreibung von Erzählungen auf „dynamisch“ angelegten Internetplattformen wie Twitter oder Facebook anwendbar sind. Puschmann/Heyd (2012) sprechen beispielsweise in Bezug auf Narrativität bei Twitter von „perversely narrative“ (ebd. 2012: 3), da bereits ,die Struktur des Programms und insbesondere die Längenbegrenzung der Entfaltung von voll ausgeformten Geschichten inhärent abträglich [ist]“" (ebd.). Im Gegensatz dazu sind beispielsweise Gründererzählungen und noch deutlicher Blogerzählungen strukturell näher an dem verortet, was man unter einer prototypischen Erzählung versteht. Daher ist es auch hier sinnvoll, formale Signale vorzustellen, die Narrativität indizieren. Auch in diesem Bereich ist es allerdings wenig zielführend, eine erschöpfende Auflistung von Hinweisen anzustreben - was m. E. auch kaum zu bewerkstelligen ist. Ich orientiere mich aus diesem Grunde an jenen Hinweisen, die für das Korpus bezeichnend sind. In den folgenden zwei Unterkapiteln nehme ich solche narrativen Merkmale in Form von charakteristisch sprachlichen Indikatoren hinsichtlich der narrativen Strukturierung (Abschn. 5.3.1) und hinsichtlich sprachlicher Verfahrensweisen (Abschn. 5.3.2) auf.

\subsubsection{Strukturelle Merkmale}

In der Erzählforschung sind ,vor allem Fragen nach der Struktur [von Erzähltexten] prägend" (Hausendorf/Quasthoff 1996: 13). Die Unterscheidung, ob man eine Erzählung oder das Erzählen untersucht, ist entscheidend für die Konzeptualisierung der narrativen Struktur. Fokussiert man die Erzählung, untersucht man die Struktur der Geschichte. Ist der Mittelpunkt des Interesses die Sprechhandlung 
,Erzählen', geht es entweder um kognitive Prozesse des Produzierens und Verstehens oder darum, eine bestimmte Art der Interaktion zu rekonstruieren. (ebd.) Die vorliegende Untersuchung befasst sich mit Erzähltexten, um die ihnen hypothetisch unterstellte Funktion nachzuverfolgen. Möchte man sich nun nicht auf eine spezifische Erzähltextstruktur festlegen, da nun mal Erzählungen von verschiedenen Internetplattformen nicht einer einheitlichen Erzählstruktur zugeordnet werden können, ist es sinnvoll sich an allgemein narrativen Strukturhinweisen zu orientieren.

Die Überlegungen zu dieser Perspektive entspringen den Ausführungen von Hausendorf/Kesselheim (2008: 91 ff.; Hausendorf et al. 2019) über narrative Strukturhinweise. Diese Betrachtungsweise erlaubt die Einordnung von Erzählfragmenten (auf Twitter oder Facebook) als narrativ, da sie dem häufig benannten Kriterium ,Abgeschlossenheit‘ oder ,lokale Begrenzung' der Geschichte entgegenstehen. Nichtsdestotrotz können einzelne Tweets aufeinander bezogen werden und sich so als Kurzbeiträge zu einer Gesamtgeschichte zusammenfügen (vgl. Texterzeugungshinweise auf Twitter in Hausendorf et al. 2018: 175). Die Autoren gründen ihren Ansatz im Allgemeinen darauf, dass in Texten Hinweise bestehen, die über die lokale Verknüpfung von Satz zu Satz hinausreichen. Dabei hat bei Erzählungen im Laufe der Zeit so eine Art von „Verselbständigung narrativer Strukturhinweise“ (Hausendorf et al. 2019: 209) stattgefunden. Sie zeigt sich in einem „Sprung von der Semantik eines bestimmten Weltbezuges zur Grammatik eines bestimmten globalstrukturellen Verknüpfungstyps“ (ebd.). Die Feststellung der Autor/innen, dass ,[d]ie Semantik der Erzählbarkeit eines Ereignisses [...] eine relativ feste Form ihres sprachlichen Ausdrucks gefunden [hat]" (ebd.), indiziert, dass neben textstrukturierenden Hinweisen weitere sprachliche Charakteristika bestehen, die uns eine Erzählung signalisieren. Wie bereits Hausendorf/Kesselheim (2008: 93) anmerken, gibt es eine Vielzahl von Typen narrativer Strukturierung, die auch ,in der Art ihrer Hinweise variieren“ (ebd.). Narrative Strukturhinweise ermöglichen es, Erzählungen unabhängig von einem festgelegten Schema zu betrachten. Auf diese Weise sind sie sozusagen modellunabhängige narrative Signale. Umgekehrt sind die unterschiedlichen narrativen Strukturhinweise verschiedenen Modellen inhärent. Im Mittelpunkt der folgenden Ausführung stehen narrative Gliederungsmerkmale nach Gülich (1976), die Hausendorf/Kesselheim (2008: 93) als narrative Strukturhinweise aufnehmen. Wie Gülich ihre Perspektive festigt, exemplifiziert ein kurzer Abriss über die damalig auftretenden Erkenntnisse und Forderungen an bestehende strukturelle Erzähltextmodellierungen. 
Mit dem Zugang zu Erzählungen über die Erzähltextstruktur haben sich neben Labov/Waletzky (1972) ebenfalls van Dijk (1969/1972) - als Vertreter der generativen Erzähltextanalyse in Anlehnung an die strukturalistische Tradition nach Propp, Bremond und Todorov - sowie Gülich (1976) - als Vertreterin des kommunikationsorientierten Ansatzes - auseinandergesetzt. Für die vorliegende Arbeit sind nach Gülichs Modell die Gliederungsmerkmale von Erzähltexten als narrative Strukturhinweise von Interesse. Daher umreise ich die Erzählgrammatik von van Dijk in absoluter Kürze, um dann auf Gülichs Modell zu sprechen zu kommen. Der kurze Abriss fokussiert van Dijks Auffassung von einer narrativen Makrostruktur, um die Unterschiede zu Gülichs terminologischer Verwendung der Makrostruktur zu verdeutlichen.

Van Dijk beschreibt eine abstrakte, den Erzähltexten zugrundeliegende Makrostruktur. Sie entspricht - wie bei Propp - einer semantischen Texttiefenstruktur. Das bedeutet, sie ist nicht mit der Tiefenstruktur der Sätze identisch, sondern sie „repräsentiert die „globale“ Bedeutung des Textes und bestimmt die semantischen Repräsentationen der Sätze und die Relationen zwischen ihnen“ (Gülich/Raible 1977: 253). Die Elemente der Makrostruktur lehnt van Dijk an die fünf Strukturelemente von Labov/Waletzky an. Allerdings ordnet er sie nicht linear - wie Labov/Waletzky - sondern hierarchisch. Obligatorische Kategorien sind bei van Dijk die Exposition, die Komplikation und die Auflösung. Dagegen betrachtet er die Evaluation und die Moral als fakultative Kategorien. Jedoch bleibt in dem Modell ungeklärt, wie sich die Oberflächenstruktur aus der Tiefenstruktur ableitet, was auch van Dijk selbst als entscheidendes noch ungelöstes Problem artikuliert. (vgl. Gülich/Raible 1977: 250 ff.) Der strukturalistische Gedanke, aus einer „begrenzten Anzahl von Unterscheidungen eine unendliche Welt von empirischen Variationen vorhersagen zu können“ (Hausendorf et al. 2019: 209), wird heute skeptisch gesehen. Doch bleibt aus den Bemühungen die Erkenntnis bestehen, „dass beim Erzählen eine Spezifik des Weltbezuges in der Grammatik natürlicher Sprachen eine relativ feste Form gefunden hat" (ebd.). Allerdings wird die reine Orientierung an der Textstruktur, insbesondere die Loslösung einer Erzählung von ihrem Kommunikationsprozess, heute nicht mehr als ausreichend betrachtet (Gülich 1996: 226). In ihrem Ansatz über eine kommunikationsorientierte Erzähltextanalyse trägt Gülich (1976) dieser Entwicklung Rechnung.

Auch wenn Gülichs Modell zeitlich etwas zurückliegt, finden sich noch heute von ihr beschriebene narrative Gliederungssignale unter den narrativen Strukturhinweisen von Hausendorf/Kesselheim $(2008,2018)$. So können diese Elemente als überzeitlich betrachtet werden. Daher wende ich sie als formale narrative Merkmale der Strukturierung auf die uRE an. Für die vorliegende Arbeit ist aus Gülichs Ansatz der Gedanke relevant, dass Erzähltexte unabhängig davon, ob sie 
schriftlich oder mündlich realisiert werden, eine Komponente in einem Kommunikationsprozess sind ${ }^{9}$. Aufgrund dessen geht die Autorin davon aus, dass sie „,rezeptionssteuernde Merkmale enthalten, an denen der Hörer [sie] als Erzähltexte erkennt" (Gülich 1976: 227). Als solche rezeptionssteuernden Merkmale stellt die Autorin verschiedene Gliederungselemente vor. Diese strukturieren eine erzählspezifische Makrostruktur formal, indem sie die Abfolge von Teiltexten ordnen. Daher wird der Begriff ,Makrostruktur' nicht wie bei van Dijk auf eine zugrundeliegende abstrakte Struktur,

„,sondern auf die Textoberfläche bezogen und definiert als die Art, die Abfolge und die Verknüpfung von funktionellen Teilen eines Textes (,Teiltexten'). Diese Teiltexte werden als Einheiten verstanden, die nicht nur inhaltlich, sondern vor allem auch formal voneinander abgrenzbar sind. Die Merkmale, die eine Gliederung des Texts in Teiltexte leisten, sollen als ,Gliederungsmerkmale" bezeichnet werden." (Gülich 1976: 242, Hervorhebung U.A.)

Die Makrostruktur eines Textes ist textsortenspezifisch. Da aber jegliche Erzähltexte in Teiltexte untergliedert sind, sind die Gliederungsmerkmale unabhängig vom narrativen Vermittlungsvorkommen auf alle Erzähltexte anwendbar und eignen sich von daher als formal narratives Merkmal. Grundsätzlich hält Gülich fest, dass ein Gliederungssignal für sich genommen noch nicht erzähltextspezifisch ist (Gülich 1976: 244). Ihrer Hypothese zufolge sind Gliederungssignale erst durch ihre Hierarchie und durch ihre Manifestationen erzähltextspezifisch. Das bedeutet, Gülich ordnet die folgenden Gliederungsmerkmale hierarchisch:

1. An erster Stelle stehen meta-narrative Sätze. Sie grenzen den Erzähltext als Ganze ab.

2. An zweiter Stelle steht die Substitution aufMeta-Ebene, ,d. h. der Verweis auf einen Teil des Texts mit einem Terminus, der dieses Textstück als erzählenden Text bezeichnet“ (Gülich 1976: 242).

\footnotetext{
${ }^{9}$ Gülichs Ansatz greift darüber hinaus deutlich weiter. Die Autorin bezieht sich auf das Modell von Gülich/Raible (1974), das - ausgehend von den Komponenten ,Sprecher ‘ und ,Hörer" - mehrere Ebenen umfasst: a) Kommunikationssituation, b) Gegenstände und Sachverhalte, c) Intention und d) Reaktion. Im Zusammenhang mit dem Kommunikationsprozess tragen die Autorin und der Autor dem Umstand Rechnung, dass ein/e Sprecher/in einem/r Hörer/in etwas mitteilt. Dahinter steht seitens der Sprecher/innen eine Intention und seitens der Hörer/innen eine Erwartung (Gülich 1976: 226). Für die Übernahme von strukturalen Merkmalen gehe ich auf den Aspekt hier jedoch nicht ein.
} 
3. An dritter Stelle stehen Episodenmerkmale. Sie sind auf Orts- und Zeitbestimmungen gerichtet, die die Gegenstände und Sachverhalte im Erzähltext entsprechend verorten.

Die Funktion meta-narrativer Sätze und der Substitution auf Meta-Ebene liegt in dem Thematisieren einer Erzählung und darin Aufmerksamkeit zu wecken (Gülich 1976: 243). In den erhobenen uRE erfüllt diese Funktion meist schon die Veröffentlichungsplattform (z. B. Corporate Blog) oder eine spezifische Rubrik (z. B. Story, Unternehmensgeschichte), die eine Geschichte fordert. Daher kommen meta-narrative Sätze in den uRE im Grunde nicht vor, mit einer Ausnahme in einem Blogbeitrag von Bosch: berichte ich nun aus dem schönen Forschungsstandort Renningen (Bosch, Trainee-Blog, 2015-12. Korpusquelle: BOSCH_502_WB). Ähnlich verhält es sich mit der Substitution auf Meta-Ebene. Sie weist den Erzähltext ,entweder als Manifestation der TextsortenKlasse ,Erzählung' insgesamt oder einer bestimmten Textsorte [... aus]“" (Gülich 1976: 244), wie beispielsweise die Eröffnung der „,autobiografischen“ Gründererzählung von Siemens: und DAS ist meine geschIchte (Korpusquelle: SIEMENS_562_WS). Als indirekte Hinweise für eine Erzählung wären Signalworte wie im folgenden Zitat denkbar: Abenteuer Afrika - Ein halbes Jahr voller kleiner Prinzen (Daimler, Das Daimler-Blog, 2014-02-19. Korpusquelle: DAIMLER_080_WB).

Episodenmerkmale untergliedert Gülich in erster Linie in Ausgangs- und Nachfolgemerkmale sowie Iterationsmerkmale. Als narratives Struktursignal für das Korpus sind insbesondere Ausgangs- und Nachfolgemerkmale von Bedeutung. Ein Ausgangsmerkmal setzt den Ausgangspunkt für die Handlung fest. Da es im Interesse der Unternehmen liegt, dass ihre uRE tatsächlich gelesen werden, ist der Erzählbeginn für die Aufmerksamkeitsweckung grundlegend. Vor diesem Hintergrund nutzen die Erzähler/innen insbesondere in Blogerzählungen eine spezifische „Erzähltechnik“, die Ausgangsmerkmale beinhaltet, wie das folgende Beispiel illustriert:

„Münster, 07.10.2014, der Wecker holte mich um 5:30 Uhr aus meinem Tiefschlaf. Es stand einiges auf dem Programm und daher machte ich mich frühzeitig und ganz nach dem Motto „,der frühe Vogel fängt den Wurm“ auf den Weg nach Bonn zur Telekom Zentrale.“(Dt. Telekom, Blog, 2014-10-09. Korpusquelle: DTKOM_835_WB)

Die Technik, in die die Ausgangsmerkmale integriert sind, ist der unvermittelte Erzählbeginn. Der Erzählbeginn gleicht einer stakkatoartigen Aufzählung von Ort, Datum und Uhrzeit. In Bezug auf solche genauen Angaben hält Gülich 
fest, dass sie weder in alltagssprachlichen noch in literarischen Erzähltexten eine gängige Praxis sind (Gülich 1976: 247). In den uRE stellt diese Möglichkeit, Ausgangsmerkmale zu platzieren, dagegen eine gängige narrative Praxis dar.

Die Ereignisdarstellung untergliedern Nachfolgemerkmale, welche sich ,auf den durch das Ausgangsmerkmal bezeichneten Zeitraum“ (ebd.: 243) beziehen. Wie beispielsweise in der folgenden Grobgliederung einer Blogerzählung von der Dt. Telekom:

„Vor ca. drei Wochen bekam ich eine Mail [...]. Die Telekom möchte mich als Blogger in die „Leave your Mark“-Kampagne 2014 ins Boot holen.

[...]

Los ging es dann am Freitag morgen in Stuttgart.

$[\ldots]$

Jetzt gab es eine wohlverdiente Mittagspause.

$[\ldots]$

Am Abend hatten wir die Möglichkeit zusammen mit einigen Telekom Managern zu grillen.“ (Dt. Telekom, Blog, 2014-09-15. Korpusquelle: DTKOM_824_WB)

Deutlich häufiger kommen jedoch Textadverbien und -konjunktionen vor, die Sätze und/oder Teiltexte zueinander in Beziehung setzen. In Bezug auf Adverbien und Konjunktionen weist Gülich allerdings darauf hin, dass sie ,nicht mehr aus dem Modell sprachlicher Kommunikation ableitbar sind“" (ebd. 1976: 243). Ihre strukturierende Funktion ist dennoch von Belang, insbesondere dann, wenn die Erzählung hinsichtlich der Zeichenmenge begrenzt ist. In diesem Fall sind sie als Strukturierungselemente mit geringem Zeichenumfang hilfreich (z. B. nachdem, jetzt, dann, anschließend u. a.). Hausendorf/Kesselheim (2008: 93) nehmen die Unterscheidung von (Gülich/Raible 1974) terminologisch auf, unterscheiden aber zwischen absoluten Episodenmerkmalen (z. B. neulich, einmal), die das Ereignis als einmalig in der Zeit verankern, und relativen Episodenmerkmalen (z. B. als, danach, kurz darauf), die die Ereignisse zueinander in ein zeitliches Verhältnis setzen. In Anbetracht der uRE von Twitter und Facebook sind Episodenmerkmale bereits im Postaufbau enthalten. Dort erkennt man einen Verbund von Beiträgen als Fragmente einer Gesamterzählung (vgl. Hausendorf et al. 2018: 173): Fest verankerte Hinweise auf das Erstellungsdatum und die -zeit ordnen Posts in eine zeitliche Abfolge und indizieren zeitliche Bezüge zwischen den Posts. Iterationsmerkmale, die wiederholte Handlungsabläufe kennzeichnen, sind in dem Korpus 
abhängig von den Themenfeldern. Handelt es sich um wiederkehrende Recruiting Veranstaltungen (z. B. Think Big oder Leave Your Mark), die Netztouren der Dt. Telekom oder gemeinnützige Aktionen, betonen Iterationsmerkmale wiederholte Unterstützungs- und Informationsangebote, wie z. B. in der Einleitung der folgenden Blogerzählung von Telefonica Dtl.:

Ganz regelmäßig reisen auch europäische Telefónica-Mitarbeiter als freiwillige Helfer nach Lateinamerika, um dort den Kindern zu helfen. (Telefonica Dtl., bolgtelefonica, 2012-12. Korpusquelle: Telef_778_WB)

Doch auch grammatische Merkmale wie das Signalwort im Plural mit Hinweisen auf eine Routinehandlung zeigen, dass sich etwas regelmäßig wiederholt. Als Beispiel folgt ein Ausschnitt aus einem audio-visuellen Tweet der Dt. Telekom zu ihrer jährlichen Netztour 2016 (Dt. Telekom, Twitter, 2016-12-27. Korpusquelle: DTKOM_847.2.1):

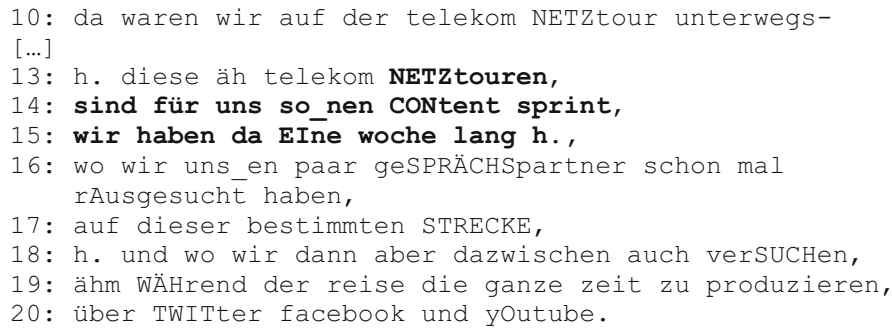

Als fünften Bestandteil der Episodenmerkmale benennt Gülich die Veränderung in der Konstellation der Handlungsträger/innen. Sie ist dann gegeben, wenn

„eine neue Person auftritt, oder wenn eine Person in einem Teiltext nicht an der Handlung beteiligt ist, oder auch wenn die Handlung in den einzelnen Teiltexten von verschiedenen Personen ausgeht.“ (Gülich 1976: 243)

Das strukturierende Element ist vor allem im Zusammenhang mit Tweets von Bedeutung. Beziehen sich mehrere Tweets auf eine gemeinsame Geschehensdarstellung (= diskontinuierliche Erzählung), dann können sich durchaus mehrere Personen als Handlungsträger/innen an und in dem Erzähltext beteiligen (siehe z. B. Abschn. 9.4). Sie geben sich in diesen Fällen entweder mit ihrem Klarnamen oder mit einem Nutzernamen zu erkennen, andernfalls wäre die Kohärenz 
zwischen den einzelnen Erzählelementen gefährdet. Das ist in diesem Falle möglich, da Unternehmen eine Verknüpfung mit den Tweets anderer Nutzer/innen vollständig vermeiden. Hausendorf et al. (2018: 179) nennen das Vorgehen eine „hermeneutische Praxis“.

Formale Merkmale einer Erzählung sind jedoch nicht ausschließlich auf die Struktur zu reduzieren. Es bestehen darüber hinaus spezifisch narrative Merkmale, die sich ebenfalls aufgrund ihrer sprachlichen Umsetzung erfassen lassen. Auch in diesem Fall beschränke ich mich auf drei charakteristische Merkmale in den erhobenen uRE.

\subsubsection{Subjektivierende Merkmale}

Eine grundlegende Unterscheidung zwischen einer Erzählung und einem Bericht ist nach Rehbein (1984: 97, zitiert nach Kern 2000: 102), dass der Bericht eine „handlungskategorisierende Tätigkeit“ ist, über die ein gemeinsamer „Wissensraum“ (ebd.) etabliert wird. Im Gegensatz dazu geht es bei Erzählungen darum, einen gemeinsamen „Vorstellungsraum“ (ebd.) zu evozieren. Daher sind Erzählungen von Subjektivität und nicht von Objektivität geprägt. Unter dieser Voraussetzung stehen die drei folgenden formalen Merkmale. $\mathrm{Zu}$ solchen subjektivierenden sprachlichen Verfahrensweisen, zählen in den uRE das Herstellen einer ,Atmosphäre', ,Gedankeneinblicke' und ,Emotionalität". Auch wenn es im ersten Moment paradox erscheint, dass ich Subjektivitätsmerkmale als formale Merkmale heranziehe, erhält die Sichtweise dadurch ihre Berechtigung, dass sie formal anhand ihrer Versprachlichung nachvollziehbar sind. Der Zugang ist somit durch Angaben von bestimmten Parametern präzisierbar. Des Weiteren stehen die drei Merkmale in enger Beziehung zu den zuvor beschriebenen inhaltlichen Merkmalen. Für diese Untersuchung ist es jedoch nicht zielführend einem inhaltlichen Merkmal konkret ein formales zuzuordnen. So kann beispielsweise der Ausdruck von Emotionen die Versprachlichung einer Evaluation sein. Ebenfalls kann er die Erfahrungshaftigkeit wie auch etwas sozial relevant Außerordentliches kommunizieren oder zur Beschreibung einer Zustandsveränderung herangezogen werden. Das verdeutlicht, dass Emotionalität, Atmosphäre und Gedankeneinblicke variable formale Merkmale darstellen. Sie dienen zwar der Operationalisierung inhaltlicher Merkmale müssen aber aufgrund ihrer vielseitigen Anwendbarkeit flexibel handhabbar bleiben. Im Folgenden stelle ich die drei Merkmale vor. Auch wenn es sich hierbei um eine theoretische Ausführung handelt, veranschauliche ich sie zu Teilen mit Datenbeispielen, um ihre Spezifik in dem jeweiligen Kontext verständlich zu machen. 
Der Ausdruck ,Atmosphäre‘ findet sich in sehr unterschiedlichen Kontexten wie z. B. in ästhetischen Diskursen über Kunstwerke, in politischen Diskursen über Verhandlungen oder in der Alltagssprache zur Personencharakterisierung (jmd. umgibt eine geheimnisvoll Atmosphäre). Mit Atmosphäre erfassen die Sprecher/innen ,etwas Unbestimmtes, schwer Sagbares“ (Böhme 2013: 21). In der Alltagssprache verfügen wir über ein reichhaltiges Vokabular, anhand dessen wir Atmosphären in Bezug auf Menschen, Räume oder die Natur näher bestimmen (bspw. heiter, gespannt, erhebend, einladend u. a.). Die Unbestimmtheit von Atmosphären resultiert hierbei nicht unbedingt aus der häufigen Verwendung, sondern aus ihrem ontologischen Status:

„Man weiß nicht recht, soll man sie den Objekten oder Umgebungen, von denen sie ausgehen, zuschreiben oder den Subjekten, die sie erfahren. Man weiß auch nicht so recht, wo sie sind. Sie scheinen gewissermaßen nebelhaft den Raum mit einem Gefühlston zu erfüllen.“ (Böhme 2013: 22)

Ausgehend von der Ästhetik hat Atmosphäre ,mit der Beziehung von Umgebungsqualitäten und menschlichem Befinden zu tun" (ebd.). Und genau darum geht es, wenn ein/e Erzähler/in in den erhobenen Daten in seiner/ihrer uRE eine Atmosphäre herzustellen versucht. Dazu imaginiert er/sie eine Stimmung, die sich auf eine Umgebung, Menschen in der Umgebung und/oder auf bestimmte Objekte richtet. Es geht um die situative Vermittlung einer Empfindungsqualität, die der/die Erzähler/in in Verbindung mit der Umgebungen oder bestimmten Objekten erfährt. Hierin liegt ein spezifisches Merkmal der Vermittlung von ,Atmosphäre', nämlich eine bewusste sprachliche Gestaltung, um die Rezipient/innen affektiv anzusprechen. Es handelt sich demnach um eine spezifische Form der Inszenierung. Das Herstellen von Atmosphäre ist der Versuch der Erzählenden, ihre Rezipient/innen in das Erzählte einzubinden und ,hineinzuziehen“. Das gelingt am ehesten, wenn die sprachlichen Trigger an bestimmtes „Weltwissen“ der Rezipient/innen andocken, um entsprechende Vorstellungen hervorzurufen. Die sprachliche Gestaltung einer Atmosphäre geht in den meisten Erzähltexten Hand in Hand mit dem Versuch einer literarischen Ästhetisierung. Zur Verdeutlichung folgende Beispiele:

(Bsp. 1) „Die Straßen sind dieselben, die Dörfer und Altstädte, die aufgereihten Zypressen, die Sonne, die in jeder Kurve durch die Blätter der zeitlosen Wälder schimmert [...]. Die Menschen stehen wie damals am Straßenrand, winken, rufen „forza“. Natürlich besonders gerne bei roten Autos.“ (Daimler, Das Blog, 2014-05-16. Korpusquelle: DAIMLER_088_WB) 
(Bsp. 2) „Der Schatten des Fernsehturms erobert das Dach des Nachbarhauses, als wir Dannys Wohnung in Mitte betreten. Die kleine Dreizimmerwohnung präsentiert sich stilsicher und elegant mit einem Schuss Unterstatement. Bücher liegen in rechten Winkeln auf Tischen, Gläser brechen das einfallende Licht, Pflanzen färben die Optik gekonnt ein - die Handschrift von Dannys Partner Andy, einem Art Director, der in Australien beim Fernsehen gearbeitet hatte. Ich traue mich kaum, das Arrangement mit meiner Anwesenheit durcheinander zu bringen, doch dann setzte ich mich auf die Couch zu Danny Solution Manager bei Eon.“ (E.ON, \#onedaywith, o. J. Korpusquelle: E.ON_637_WB)

(Bsp. 3) „Die Wolken hängen tief über Frankreich. Der Flugzeugmotor dröhnt während unseres rasanten Sturzflugs. Mein linker Arm wird bedrohlich heiß. Ich bin nicht angeschnallt - natürlich nicht! Das Cockpit vibriert und ist so eng, dass ich immer wieder aufpassen muss, dem Abgasrohr direkt links neben mir nicht zu nahe zu kommen. Der Pilot sitzt Schulter an Schulter rechts von mir, trägt Fliegerbrille und gibt mir im bollernden Lärm Zeichen, auf den Benzindruck zu achten. Ich greife hinter seinen Sitz und pumpe ein paar Mal an einem Hebel mit Holzgriff, um den Druck auf der antik wirkenden Skala wieder hoch auf 1 bar zu bringen, so wie man es mir vorm Start beigebracht hat.“ (Daimler, Das Blog, 2014-05-19. Korpusquelle: DAIMLER_087_WB)

Die sprachlich inszenierte Atmosphäre benötigt Raum, um die Qualität des Empfindens zu transportieren. Daher zeichnen sich entsprechende Erzähltextsequenzen dadurch aus, dass sie verhältnismäßig umfangreiche Deskriptionen in Form ausschweifender Passagen enthalten. In diesen Beispielen bezieht sich das Atmosphärische in Bsp. 2 auf die Empfindungen des Autors in bestimmten Räumlichkeiten, die sinnbildlich für eine Personencharakterisierung stehen, und in Bsp. 3 auf die Empfindungen des Autors in Bezug auf ein bestimmtes Objekt. Sprachliche Kennzeichen finden sich in Adjektiven und Phrasen, die mit möglichst eingängigen Sinneseindrücken assoziierbar sind. In den Beispielen sind es visuelle (einfallende Licht, Pflanzen färben die Optik), auditive (Flugzeugmotor dröhnt, bollernden Lärm) und taktile Eindrücke (Arm wird bedrohlich heif, das Cockpit vibriert). Hinzu kommt das Erzeugen von Vorstellungsbildern (Wolken hängen tief, antik wirkenden Skala, ist so eng, Bücher liegen in rechten Winkeln auf Tischen), die dadurch entstehen, dass die beschriebenen Sinneseindrücke in allseits bekannte Empfindungen integriert werden sollen (z. B. Ehrfurcht, Abenteuerlust), um so die Vorstellungskraft der Rezipient/innen zu befeuern. Dadurch kommt es zu einer Ansammlung von Eindrücken, um eine bestimmte Atmosphäre zum erzählten Zeitpunkt greifbar zu machen. Wird eine Erzählung audiovisuell dargeboten, kommen zu den sprachlichen Kennzeichen der Ton und das (Bewegt-) Bild. Hinsichtlich des Tons sprechen Lahn/Meister (2013: 266) von der „Klangatmosphäre“, die durch Stimmen, Geräusche und (Hintergrund-)Musik entsteht. 
Die Kombination von Bewegtbild und Musik parallelisiert Stimmungsindizien, die sich zu einem möglichst eindeutigen Stimmungsbild verdichten. Das bedeutet, dass sich alle Komponenten bis ins Detail an der gewünschten Vermittlung von Atmosphäre ausrichten: Settings, Farben, Kameraeinstellungen, Personen und ihre Performanz, Utensilien u. a.

Das zweite formale Kennzeichen, welches in den erhobenen uRE auf Narrativität hinweist, ist die sprachliche Realisierung von Gedankeneinblicken. Darüber gewähren Erzählende ihren Rezipient/innen Einblicke in ihre mentalen Vorgänge. In den uRE sind Gedankeneinblicke affektiv mit der erzählten Welt oder erzählten Situation verbunden. Umsetzen können sie die Erzähler/innen in Form des Gedankenzitats und des Inneren Monologs. (vgl. Martinez/Scheffel 2007: 62) Anwendung finden Gedankeneinblicke charakteristischerweise, um spontane Gedankenäußerungen und innere „Selbstgespräche“ in die uRE einzubringen. Formal sprachliche Merkmale sind die markierten und/oder unmarkierten Redewiedergaben aus der Perspektive der Erzählenden. In den erhobenen uRE leiten sie für gewöhnlich ein Exklamativsatz oder Interogativsatz ein. Exklamativsätze symbolisieren kurzfristig auftretende Gedanken, die der/die Erzähler/in durch den Kopf schießen:

„Ich bin nicht angeschnallt - natürlich nicht!“ (Daimler, Das Blog, 2014-05-19. Korpusquelle: DAIMLER_087_WB)

Interrogativsätze leiten dagegen für gewöhnlich eher eine Reflexion ein:

„Wie wurde es nach 20 Jahren erweckt? Was war der Anlass dazu? Hmm..., ich kann es nicht genau beantworten.“ (Daimler, Das Blog, 2015-11-25. Korpusquelle: DAIMLER_057_WB).

Gedankeneinblicke mittels des Inneren Monologs oder eines Gedankenzitats unterscheiden sich darin, dass ein Innerer Monolog ohne Inquit-Formel realisiert wird wie in den obigen zwei Beispielen. Dadurch entsteht der Eindruck einer spontanen inneren Äußerung, da die Rede ,sich gewissermaßen selbst [spricht] (Lahn/Meister 2013: 123). Das Gedankenzitat weist sich anhand einer Inquit-Formel aus wie in den folgenden zwei Beispielen:

(Bsp. 1),„, Warum ausgerechnet Israel?““, dachte ich [Inquit-Formel], und zögerte, als die Kollegen aus Tschechien mit der Bitte auf mich zukamen, eine JournalistenReise nach Tel Aviv zu organisieren.“ (Dt. Telekom, Weblog, 2016-11-07. Korpusquelle: DTKOM_837_WB) 
(Bsp. 2) „Mensch, äh Auto, denke ich [Inquit-Formel], nun freu dich doch, es geht wieder los. So wie früher. Nur wir zwei und der einsam, endlos lange Highway vor uns. Wo sind wir nicht schon überall zusammen gewesen?“ (Daimler, Das Blog, 201409-22. Korpusquelle: DAIMLER_079_WB)

Ist eine Gedankenrede als direkte Rede markiert, wird die gewünschte Unmittelbarkeit dadurch evoziert, dass der Eindruck erzeugt wird, es handle sich um eine möglichst authentische - im Sinne einer wortgetreuen - Wiedergabe des innerpsychischen Vorgangs. Kennzeichnend für Gedankeneinblicke ist in den Daten, dass sie in der Ich-Form formuliert werden. Da es sich um faktuale Erzählungen handelt, können sie nicht auf andere Aktanten in der uRE ausgelagert werden (siehe hierzu z. B. Abschn. 9.2.5.2).

Bei dem Merkmal ,Emotionalität' geht es in der alltagsweltlichen Konzeptualisierung um den Ausdruck von Gefühlen. Emotion und Gefühl werden synonym verwendet. Die Perspektive wird teilweise in linguistischen Arbeiten übernommen, als die Verfasser/innen keine explizite Unterscheidung treffen (Fiehler 1990; Hermanns 1995; Stoeva-Holm 2005). Differenzieren die Autor/innen, dann vertreten sie unterschiedliche Sichtweisen auf den Gegenstand (Wierzbicka 1999; Fries 2004). (vgl. Schwarz-Friesel 2007: 139) Im Umgang mit dem formalen Merkmal ,Emotionalität" verwende ich die Termini Emotion und Gefühl synonym wie in der alltagsweltlichen Perspektive.

In der mündlichen Kommunikation von Emotionen unterscheiden LuciusHoene/Deppermann (2004: 39) zwischen beabsichtigter Re-Inszenierung eines Affekts und dem tatsächlichen Erleben. Bei einer beabsichtigen Re-Inszenierung handelt es sich um eine kontrollierte, hörerbezogene Gestaltung, die die Erzähler/innen unter strategischen Gesichtspunkten gestalten. Sie ist nachvollziehbar „durch die Nutzung konventioneller emotionaler Ausdrucksmittel“ (ebd.), die die Erzähler/innen strategisch auswählen. Die Kommunikation von Emotion kann durch die Art des Thematisierens eines Erlebens oder einer Emotion nachvollzogen werden. Fiehler (2001) benennt vier Verfahren, die das ermöglichen: a) die begriffliche Erlebensbenennung anhand eines Erlebenswortschatzes, b) Erlebensbeschreibungen, c) das Benennen und Beschreiben von erlebensrelevanten Ereignissen und Sachverhalten sowie letztlich d) das Erzählen oder Beschreiben selbst von situativen Umständen, in deren Rahmen ein Erleben stattfand. (ebd.: 1430 ff.) Im Regelfall zeigen Sprecher/innen ihre Emotionen bereits schon durch die Art und Weise an, wie sie sich über ein Thema äußern. Hierfür können Emotionen durch die a) Anordnung der Ereignisdarstellung selbst zum Ausdruck kommen. Die Auswahl der erzählten Elemente und die Abfolge der 
Ereignissegmente suggerieren bereits eine bestimmte Bedeutsamkeit (vgl. LuciusHoene/Deppermann 2004: 21). Zusätzlich manifestieren sich Emotionen anhand b) nonvokal nonverbaler Merkmale wie z. B. graphische Ikonizität (Emoticons, Groß- oder Fettschrift u. a.), Mimik oder Gestik sowie c) die Gesprächsmodalität (z. B. ironisch, lustig, traurig u. a). Gerade im Zusammenhang mit Emotionen weist Fiehler darauf hin, dass das Erzählen in weiten Teilen eine „emotionsintensive kommunikative Aktivität" (Fiehler 2001: 1436) ist. Die verbale/schriftliche Darstellung von Emotionen lässt sich lexikalisch bspw. anhand eines bestimmten Erlebenswortschatzes (Fiehler 2001: 1431) und syntaktisch durch Vergleiche (Schwarz-Friesel 2007: 190) oder Metaphern (vgl. Schwarz-Friesel 2007: 199; Fiehler 2001: 1431) ausdrücken. Dabei müssen re-inszenierte Emotionen nicht zwingend mit den Emotionen der Erzählenden zum Zeitpunkt des Erzählens übereinstimmen (vgl. Lucius-Hoene/Deppermann 2004: 39). Da gerade in schriftlichen Erzähltexten Emotionen irgendwie kenntlich gemacht werden müssen, handelt es sich bei der Re-Inszenierung um eine gängige narrative Praxis. Das tatsächliche Erleben zeichnet sich durch ein „Vereinnahmt-Werden durch einen Affekt" (ebd.) aus. Die Emotionen treten im Erzählprozess auf, während die Erzähler/innen Ereignisse rekapitulieren. Das ist - in Bezug auf die uRE - ein Sonderfall, der audio-visuelle Erzählungen betrifft. Hierbei spielt die Performanz eine herausragende Rolle, also die Art und Weise, wie die Erzähler/innen kommunizieren. Die Kombination von Wort und visueller Darbietung soll in den uRE Emotionen nicht nur benennen, sondern „erlebbar“ machen. Aufgrund der Untersuchungsergebnisse über den ,figurierten Erzähler (Abschn. 7.2.2.1) lässt sich festhalten, dass es sich hierbei um eine Mischung aus tatsächlich empfundenem und eingeübtem bzw. verstärkt inszeniertem Emotionsausdruck handelt.

\subsection{Verortungsschema: Grundanforderungen + Indikatoren narrativer Praktiken}

Basierend auf den vorausgegangenen Ausführungen lässt sich ein datengeleitetes, objektbezogenes Verortungsschema erstellen, anhand dessen sich die analysierten uRE als narrativ legitimieren. Die in den letzten zwei Kapiteln ausgeführten inhaltlichen und formalen Merkmale verstehe ich im Zusammenhang mit dem folgenden Verortungsschema als narrative Indikatoren, da Erzählungen im Unternehmenskontext eher selten - wenn überhaupt - einer erzähltheoretischen Reflexion unterliegen. Damit ist verbunden, was Norlyk et al. (2014) wie folgt konstatieren: „The term 'story' does not follow the traditional narratological definition of a story“ (ebd.: 105). In letzter Konsequenz folgt daraus, dass 
erzähltheoretische Modelle, Konzepte und Theorien nicht in Gänze und insbesondere nicht in einer Modellierung auf alle erhobenen Daten zu gleichen Teilen anwendbar sind.

Ich orientiere mich deswegen an dem Vorgehen von Martinez (2017), um ein objektbezogenes narratives Bezugssystem für die uRE bereitzustellen, das sie gleichzeitig anhand ihrer Spezifik beschreibt. Das Verortungsschema ist in erster Linie dazu entworfen worden, um mithilfe der Merkmale die untersuchten uRE von anderen Darstellungsformen (z. B. Berichten, Argumentieren) zu differenzieren. Martinez beginnt seinen Ansatz mit der obligatorischen Minimalbedingung der ,Geschehensdarstellung‘. Diese übernehme ich mit leicht anderer Gewichtung, die den Veröffentlichungsplattformen geschuldet ist, und formuliere daraus die erste Grundvoraussetzung von zweien. Die zweite datengeleitete Grundvoraussetzung bezieht sich auf die Träger/innen der Erzählaktivität. Die beiden Grundvoraussetzungen erweitere ich mit den zuvor beschriebenen inhaltlichen und formalen narrativen Indikatoren. Dabei entsprechen die inhaltlichen Merk-

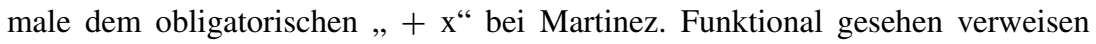
sie darauf, die uRE als erzählenswert auszuweisen. Die Erweiterung um formale Merkmale entstammt charakteristisch sprachlichen Indikatoren der uRE. Zusammen mit den zwei Grundvoraussetzungen entsteht daraus das Verortungsschema. Die folgende Tabelle zeigt die Grundelemente des Schemas, die ich anschließend erläutere (Abb. 5.1):

\begin{tabular}{c}
\hline Grundvoraussetzungen \\
\hline a) Geschehensdarstellung: Adaption der Minimalanforderung nach Martinez (2017) \\
\hline b) Äußerungsproduktion: Träger/innen der Erzählaktivität \\
\hline Narrative Indikatoren \\
$\begin{array}{c}\text { Optional } \text { inhaltliche } \text { Merkmale } \\
=\text { optionale , }+\mathrm{x}^{\prime} \text { bei Martinez }\end{array}$ \\
\hline
\end{tabular}

Abb.5.1 Grundelemente des Verortungsschemas für die erhobenen uRE

Die Grundvoraussetzungen an Erzählungen sehe ich hinsichtlich der Daten in den zwei Bereichen: a) Geschehensdarstellung und b) Äußerungsproduktion. In Bezug auf die Geschehensdarstellung orientiere ich mich an den Minimalanforderungen nach Martinez (2017), deren Bestimmungselemente ich aufgrund des Erhebungskontextes zu Teilen anders gewichte. Ursächlich dafür sind die technischen Gegebenheiten der Internetplattformen, die die Geschehensdarstellung 
beeinflussen. Martinez legt bewusst nicht fest, mithilfe welcher semiotischen Ressource die Geschehensdarstellung erfolgt. Somit ist der Einbezug von Fotografien und Bewegtbildern in das Korpus möglich. Der Autor formuliert die drei grundlegenden Merkmale der Darstellung: Konkretheit, Temporalität und Kontiguität. Das Merkmal ,Konkretheit' bezieht sich bei Martinez auf die Darstellung von singulären, konkreten Gegenständen und Sachverhalten. In den Daten ist jedoch der Merkmalsaspekt ,singulär' nicht immer gegeben. Beispielsweise erzählen Mitarbeiter/innen von ihrem Arbeitsalltag, der alles andere als ein singuläres Ereignis ist. Die „Daseinsberechtig“ als Erzählung erhält die Darstellung erst im Zusammenspiel mit inhaltlichen und formalen Merkmalen, die das Ereignis als narrativ exponieren. Daher schließe ich mich der Merkmalsbestimmung ,konkret' als obligatorisch an, während ich den Aspekt ,singulär' in Anbetracht der vorausgesetzten narrativen Indikatoren als nicht zwingend notwendig erachte. Das Merkmal der Temporalität bezieht Martinez auf den Zeitablauf der Geschehensdarstellung. Das Charakteristikum des zeitlichen Ablaufs ist die chronologische Abfolge von Ereignissen. Da auf den Internetplattformen Facebook und Twitter mehrere Personen an einer Erzählung mitwirken, wird dadurch die chronologische Abfolge unregelmäßig oder die Geschehensdarstellung lückenhaft. Lücken und/oder Unregelmäßigkeiten in der Abfolge von Posts oder Tweets ändern jedoch nichts daran, dass es sich um Elemente einer uRE handelt und die Rezipient/innen sie als solche erkennen können. Aufgrund der Veröffentlichungssituation im Internet steht das Merkmal ,chronologisch “ - als distinktes Merkmal - auf dem Prüfstand, insofern es offensichtlich andere Indikatoren gibt, die das Merkmal kompensieren können (z. B. inhaltliche Bezüge, Akteure u. a., vgl. Abschn. 9.4.5.1). Gewisse zeitliche Orientierungshinweise sind aber nach wie vor notwendig. Die Frage ist lediglich, ob das ausschließlich die chronologische Ereignisabfolge gewährleistet (siehe hierzu auch Page 2015; Dayter 2015; Puschmann/Heyd 2012). Das worauf die chronologische Abfolge zielt, nämlich die Verdeutlichung eines vorher und eines nachher, bleibt bestehen. Das Merkmal der Kontiguität zeichnet sich dadurch aus, dass die Ereignisse räumlich, zeitlich oder kausal aufeinander bezogen sind. Ich vertrete hier die Ansicht, dass das Merkmal den Aspekt ,chronologisch“ in Teilen auffangen kann, da es logische und inhaltliche Bezüge eröffnet, die den Rezipient/innen zur Orientierung dienen.

Die zweite Grundvoraussetzung, die ich an die uRE stelle, bezieht sich auf die Äußerungsproduktion. In der face-to-face Kommunikation produziert/produzieren für gewöhnlich eine oder mehrere Personen (vgl. Quasthoff 1980, kooperative Erzählproduktion) den Erzähltext. Wie in Abschnitt 7.1 „Text“-externe Produktionsbedingungen näher erläutert, besitzen uRE nicht immer eine/n ausgewiesene/n Erzähler/in. Dennoch benötigt eine Erzählung eine/n Träger/in der Erzählaktivität 
(Gülich 1976). In den erhobenen Daten lässt sich ein/e solche/r Träger/in entweder als Ich-Erzähler/in ausmachen, die an den erzählten Ereignissen beteiligt war (homodiegetische/r Erzähler/in) oder eine nicht direkt beteiligte neutrale Person rekapituliert in der dritten Person die Ereignisse (heterodiegetische/r Erzähler/in). Diese Personen nenne ich Erzähler/innen erster Instanz, da sie ihre uRE an die Rezipient/innen richten. Daneben gibt es auch Erzähler/innen zweiter Instanz. Das sind Personen, die im Rahmen eines Erzähltextes miteinander kommunizieren (vgl. Gülich 1976: 229).

Sind die zwei Grundvoraussetzungen erfüllt, dann erfüllen die uRE jeweils ein inhaltliches und min. ein formales Merkmal der narrativen Indikatoren, um sich als narrativ zu qualifizierten. Übertrage ich die Anforderungen in das Grundschema, sieht es wie folgt aus (Abb. 5.2):

\begin{tabular}{|c|c|c|}
\hline \multicolumn{3}{|c|}{ Grundvoraussetzungen } \\
\hline \multicolumn{3}{|c|}{$\begin{array}{l}\text { Geschehensdarstellung nach Martinez (2017) inhaltlich adaptiert: } \\
\text { a) Konkretheit: Darstellung konkreter Ereignisse und/oder Sachverhalte. } \\
\text { b) Temporalität: Es besteht ein vorher und ein nachher. } \\
\text { c) Kontiguität: Ereignisse sind räumlich, zeitlich oder kausal aufeinander bezogen. }\end{array}$} \\
\hline \multicolumn{3}{|c|}{$\begin{array}{c}\text { Äußerungsproduktion: } \\
\text { Träger/in der Erzählaktivität in erster oder zweiter Instanz }\end{array}$} \\
\hline \multicolumn{3}{|c|}{ Narrative Indikatoren } \\
\hline $\begin{array}{l}\text { Variable inhaltliche Merkmale } \\
\rightarrow \text { optionale „, }+\mathrm{x}^{\circ} \text { bei Martinez }\end{array}$ & $\rightarrow$ spezifisch & $\begin{array}{l}\text { ormale Merkmale } \\
\text { orachliche Kennzeichen }\end{array}$ \\
\hline Evaluationen & Textstrukturelle M.: & Narrative Struktursignale \\
\hline $\begin{array}{l}\text { Das sozial relevant Außergewöhnliche } \\
\text { Erfahrungshaftigkeit } \\
\text { Zustandsveränderung }\end{array}$ & Subjektivierende M.: & $\begin{array}{l}\text { Sprachlicher Aufbau von } \\
\text { Atmosphäre } \\
\text { Gedankeneinblicke } \\
\text { Emotionsausdruck }\end{array}$ \\
\hline
\end{tabular}

Abb.5.2 Grundschema zur narrativen Verortung der erhobenen uRE mit ausgewählten Indikatoren

Die narrativen Indikatoren verdeutlichen, dass Autor/innen von uRE sich auf narrative Gestaltungsmittel ,spezialisieren“, was zu der heterogenen Datenlage führt. Die Flexibilität des Verortungsschemas ermöglicht es, neben charakteristischen Erzähltexten narrative Gebilde aufzunehmen, die sich in Teilen erzählspezifische Darstellungsverfahren zu eigen machen, um narrative Praktiken von Unternehmen zur (S)D genauer zu erfassen. 
Open Access Dieses Kapitel wird unter der Creative Commons Namensnennung 4.0 International Lizenz (http://creativecommons.org/licenses/by/4.0/deed.de) veröffentlicht, welche die Nutzung, Vervielfältigung, Bearbeitung, Verbreitung und Wiedergabe in jeglichem Medium und Format erlaubt, sofern Sie den/die ursprünglichen Autor(en) und die Quelle ordnungsgemäß nennen, einen Link zur Creative Commons Lizenz beifügen und angeben, ob Änderungen vorgenommen wurden.

Die in diesem Kapitel enthaltenen Bilder und sonstiges Drittmaterial unterliegen ebenfalls der genannten Creative Commons Lizenz, sofern sich aus der Abbildungslegende nichts anderes ergibt. Sofern das betreffende Material nicht unter der genannten Creative Commons Lizenz steht und die betreffende Handlung nicht nach gesetzlichen Vorschriften erlaubt ist, ist für die oben aufgeführten Weiterverwendungen des Materials die Einwilligung des jeweiligen Rechteinhabers einzuholen.

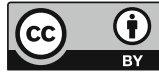

